\title{
Pathogen-Specific De Novo Antimicrobials Engineered Through Membrane Porin Biomimicry
}

Andrew Simonson, Agustey Mongia, Matthew R. Aronson, John N. Alumasa, Dennis Chan, Adam Bolotsky, Aida Ebrahimi, Elizabeth A. Proctor, Kenneth C. Keiler, Scott Medina

Submitted date: 07/05/2020 - Posted date: 12/05/2020

Licence: CC BY-NC-ND 4.0

Citation information: Simonson, Andrew; Mongia, Agustey; Aronson, Matthew R.; Alumasa, John N.; Chan, Dennis; Bolotsky, Adam; et al. (2020): Pathogen-Specific De Novo Antimicrobials Engineered Through Membrane Porin Biomimicry. ChemRxiv. Preprint. https://doi.org/10.26434/chemrxiv.12267992.v1

Precision antimicrobials that can kill pathogens without damaging host commensals hold potential to cure disease without antibiotic-associated dysbiosis. Here we report the de novo design of host defense peptides that have been rationally engineered to precisely target specific pathogens by mimicking key molecular features of the target microbe's unique channel-forming membrane proteins, or porins. This biomimetic strategy exploits physical and structural motifs of the pathogen envelope, rather than targeting resistance-susceptible protein biochemical pathways, to construct fast-acting precision bacteriolytics. Utilizing this approach, we design an antitubercular sequence that undergoes instructed, tryptophan-zippered assembly within the mycolic-acid rich outer membrane of Mycobacterium tuberculosis (Mtb) to specifically kill the pathogen without collateral toxicity towards lung commensals or host tissue. These mycomembrane-templated mechanisms are rapid and synergistically enhance the potency of antibiotics that otherwise poorly diffuse across the rigid Mtb envelope, particularly those that exploit porins for antimycobacterial activity. This new porin-mimetic paradigm may serve as a conceptual basis for the directed design of new narrow-spectrum antimicrobial scaffolds.

File list (1) 


\title{
Pathogen-specific de novo antimicrobials engineered through membrane porin biomimicry
}

Andrew W. Simonson ${ }^{1}$, Agustey S. Mongia ${ }^{1}$, Matthew R. Aronson ${ }^{1}$, John N. Alumasa ${ }^{2}$, Dennis C. Chan $^{1,3,4}$, Adam Bolotsky ${ }^{5,6}$, Aida Ebrahimi ${ }^{1,5,6,7}$, Elizabeth A. Proctor ${ }^{1,3,4}$, Kenneth C. Keiler $^{2}$ \& Scott H. Medina ${ }^{1, *}$

${ }^{1}$ Department of Biomedical Engineering, Penn State University, University Park, PA, USA.

${ }^{2}$ Department of Biochemistry and Molecular Biology, Penn State University, University Park, PA, USA.

${ }^{3}$ Departments of Neurosurgery and Pharmacology, Penn State College of Medicine, Hershey, PA, USA.

${ }^{4}$ Center for Neural Engineering, Penn State University, University Park, PA, USA.

${ }^{5}$ Department of Materials Science and Engineering, Penn State University, University Park, PA, USA.

${ }^{6}$ Materials Research Institute, Penn State University, University Park, PA, USA.

${ }^{7}$ Department of Electrical Engineering, Penn State University, University Park, PA, USA.

\begin{abstract}
Precision antimicrobials that can kill pathogens without damaging host commensals hold potential to cure disease without antibiotic-associated dysbiosis. Here we report the de novo design of host defense peptides that have been rationally engineered to precisely target specific pathogens by mimicking key molecular features of the target microbe's unique channel-forming membrane proteins, or porins. This biomimetic strategy exploits physical and structural motifs of the pathogen envelope, rather than targeting resistance-susceptible protein biochemical pathways, to construct fast-acting precision bacteriolytics. Utilizing this approach, we design an antitubercular sequence that undergoes instructed, tryptophan-zippered assembly within the mycolic-acid rich outer membrane of Mycobacterium tuberculosis (Mtb) to specifically kill the pathogen without collateral toxicity towards lung commensals or host tissue. These mycomembrane-templated mechanisms are rapid and synergistically enhance the potency of antibiotics that otherwise poorly diffuse across the rigid Mtb envelope, particularly those that exploit porins for antimycobacterial activity. This new porin-mimetic paradigm may serve as a conceptual basis for the directed design of new narrow-spectrum antimicrobial scaffolds.
\end{abstract}




\section{Introduction}

Small molecule antibiotics are currently our best weapons against bacterial infections. However, their overuse has led to the widespread emergence of multidrug resistance (MDR) and, combined with a lagging discovery pipeline, we now find ourselves quickly running out of useful drugs. An underappreciated, but important, contributor to resistance is the human microbiome. ${ }^{1}$ Repeated antibiotic use promotes the accumulation of resistance genes in the overexposed commensal microflora which can then horizontally transfer these genes to colonizing pathogens. Emerging evidence now suggests that population networks enables the exchange of these genes across vast geospatial distances, leading to a globally connected human resistome. ${ }^{2,3}$ In the individual, these commensal communities also play important roles in maintaining immune and metabolic homeostasis, as well as preventing opportunistic infections. ${ }^{4,5}$ Hence, not only do our microbiomes serve as a long-term reservoir of resistance genes that encourage MDR infections, but their disruption by broad-spectrum drugs has immediate and significant health effects. Recognizing the incalculable benefits broad-spectrum antibiotics have had on human health, it is clear we must develop pathogen-specific antimicrobials that can minimize, or altogether avoid, the use of our remaining antibiotic arsenal so as to preserve the protective human microbiome, enhance patient outcomes and reduce the spread of resistance.

Traditional approaches for the discovery of narrow-spectrum antimicrobials relies on large empirical compound screens. These discovery methods are costly, laborious and hindered by the need for extensive med-chem optimization of lead candidates. ${ }^{6,7}$ Further, a major focus of these campaigns is the identification of new compounds with unique protein targets. Yet, where specific proteins are involved the possibility exists for genetic mutations and bacterial resistance. An alternative strategy is to rationally design antimicrobials that do not target a particular biochemical pathway, but instead act by mimicking physical features of the target pathogen envelope that are difficult for the microbe to mutationally alter. Utilizing native cell wall biomolecules as templates, this approach would allow for the development of biomimetic 'Trojan horse' antimicrobials that undergo templated assembly within the target microbial envelope to enable precision killing of pathogens with minimal off-target effects.

Here, we apply this strategy to design pathogen-specific antimicrobial host defense peptides against Mycobacterium tuberculosis (Mtb), the causative agent of Tuberculosis (TB). This is achieved by rationally engineering de novo peptides to assemble into structures mimicking the mycobacterial specific cell wall porin, MspA. Complimentary atomistic simulations, biophysical analysis and microbiologic assays demonstrate that membrane lysis by the mimetic peptide, named myco-membrane associated disruption 1 (MAD1) sequence, is selectively potentiated upon interaction with the mycolic-acid rich outer mycobacterial surface. This enables MAD1 to elicit rapid and selective killing of Mtb (death occurs in $<1$ hour) without collateral toxicity towards polymicrobial respiratory commensals and host lung tissue. Further, these membranespecific mechanisms synergistically enhance the potency of clinical TB antibiotics that otherwise poorly diffuse across the rigid outer myco-membrane of Mtb pathogens.

\section{Results}

\section{Biomimetic design of MAD1}



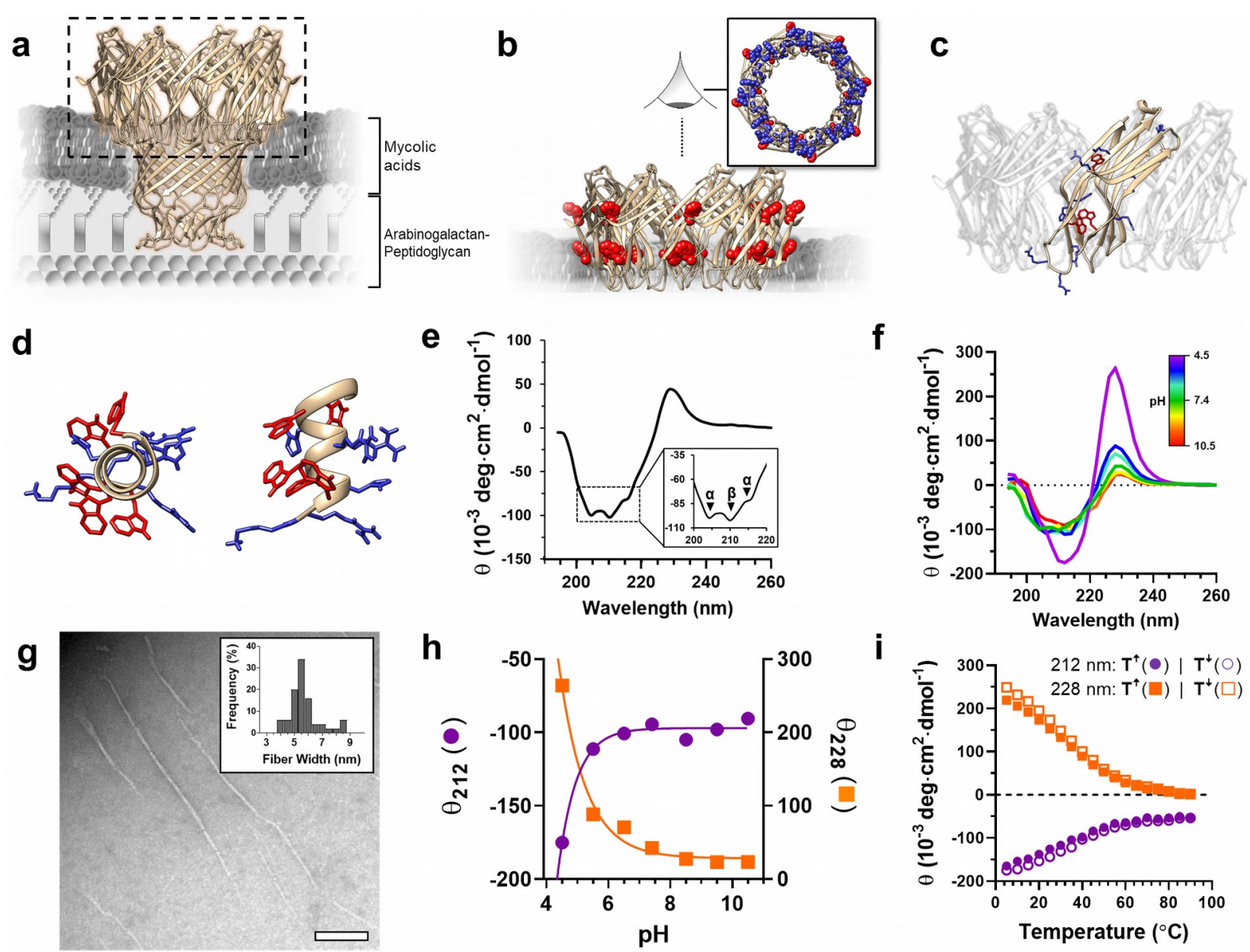

Fig. 1 | Biomimetic design and biophysical analysis of MAD1. a, Model of the MspA porin docked in a rendering of the Mtb envelope (PDB structure 1UUN). b, Concentric rings of tryptophan residues (red) circumscribing the porin head is key to MspA's ability to anchor at the mycolipid-water interface. Inset: Model of MspA viewed from above (red $=$ tryptophan, blue = basic residues). $\mathbf{c}$. Highlighted protein sub-unit of the MspA rim domain. d, Minimized model of a MAD1 helix (left: axial view; right: side view; red = tryptophan, blue = basic residues). The peptide mimics the facially amphiphilic character and extended terminal basic residues of the MspA rim subunit. e, f, Circular dichroism spectra of the MAD1 peptide at (e) physiologic $\mathrm{pH}(7.4$; inset magnifies signal from $200-220 \mathrm{~nm}$ ) or (f) as a function of decreasing $\mathrm{pH}$ from 10.5 (red) to 4.5 (purple). g, TEM micrograph of MAD1 nanofibrilar assembles (pH 4.5). Scale bar $=100 \mathrm{~nm}$. Inset: Histogram of lateral fibril width $(\mathrm{n}=50) . \mathbf{h}, \mathrm{pH}$-dependent change in the molar ellipticity of MAD1 at $228 \mathrm{~nm}(\boldsymbol{\square}$, orange) or $212 \mathrm{~nm}(\bullet$, purple). i, Temperature-dependent change in MAD1 molar ellipticity at $228 \mathrm{~nm}$ (circles, orange) or $212 \mathrm{~nm}$ (triangle, purple). Closed symbols represent temperature ramp up, and open symbols the reverse temperature ramp down. $\mathrm{n} \geq 3$ per experiment, with representative micrographs or plots shown.

The design of MAD1 was inspired by the mycobacterial-specific porin MspA. This $\beta$-sheet rich, homo-octameric transmembrane protein is unique to pathogenic mycobacteria and forms a single-channel pore that permits the diffusion of hydrophilic molecules across the Mtb outer membrane (Fig. 1a). ${ }^{8}$ Importantly, the structure of MspA has evolved to assemble within the distinct environment of the Mtb cell envelope, which is defined by an extremely rigid outer membrane that is rich in mycolic acids. Notably, MspA presents a distinctive girdle of tryptophan residues in its membrane-spanning domain that has been specially adapted to localize the porin rim at the mycolipid-water interface (Fig. 1b). ${ }^{9}$ These tryptophan-rich features are unique to MspA and are not found in other porins identified in Gram-negative bacteria. ${ }^{8}$ Juxtaposed to this nonpolar outer porin surface is a hydrophilic interior that is rich in basic residues. In addition, arginines 33, 35 and 38 in the subunit loop region extend into the mycolic-peptidoglycan 
interface (Fig. 1c), suggesting they support anchorage of the porin within the mycolylarabinogalactan-peptidoglycan complex. ${ }^{10}$ To replicate these molecular features in a host defense peptide, we designed the thirteen amino acid de novo sequence MAD1: KRWHWWRRHWVVW-NH . Molecular simulations suggest the peptide adopts an alpha helix defined by a nonpolar tryptophan-rich domain opposed to a charged polar surface (Fig. 1d), thus recapitulating the facial amphiphilicity of MspA. In addition, MAD1's N-terminal lysine and arginine residues adopt extended side-chains analogous to the basic loop of the porin rim subunit (Fig. 1c).

Important to MAD1's bioactivity are two histidine residues displayed on its polar face. These histidines enable pH-triggered assembly of MAD1 within the acidic phagolysosome of macrophages ( $\mathrm{pH} 5.5$ - 6.0), ${ }^{11}$ a key cellular niche for Mtb pathogens during early TB infection. ${ }^{12,13}$ To demonstrate this, circular dichroism (CD) spectroscopy was performed under various pathophysiological conditions. At $\mathrm{pH} 7.4$, MAD1 displays a CD spectrum suggestive of mixed $\alpha$-helical (minima at $206 \mathrm{~nm}$ and $219 \mathrm{~nm}$ ) and $\beta$-sheet (minima at $212 \mathrm{~nm}$ ) conformations (Fig. 1e, see inset). An exciton band at $228 \mathrm{~nm}$ is indicative of interactions between tryptophan aromatic indole chromaphores. ${ }^{14}$ TEM micrographs show that at pH 7.4 MAD1 organizes into short fibrils that are $100-200 \mathrm{~nm}$ in length and $\sim 6 \mathrm{~nm}$ in width (Supplementary Fig. 1). Taken together, this suggests that MAD1 helices, estimated to be $\sim 1.7 \mathrm{~nm}$ in the axial dimension, undergo Trp-Trp intermolecular pairing at physiologic $\mathrm{pH}$ to form laminated proto-fibrillar assemblies. Under acidic conditions, however, MAD1 evolves an unusual CD spectrum with intense exciton-coupled bands at 212 and $228 \mathrm{~nm}$ (Fig. 1f), a profile analogous to a putative tryptophan zipper. ${ }^{14,15}$ More importantly, this spectrum closely matches that of the full-length recombinant MspA protein, reported to display similar exciton bands at 216 and $230 \mathrm{~nm} .{ }^{16}$ TEM imaging reveals that under these conditions MAD1 zippers into monomorphic amyloid-like fibers approximately $300-700 \mathrm{~nm}$ in length and $5.5 \mathrm{~nm}$ in width (Fig. 19). Collectively, our data at both $\mathrm{pH} 7.4$ and 4.5 indicate that MAD1 undergoes supramolecular assembly to form tryptophan-zippered architectures. Importantly, these higher-order assemblies mimic the $\beta$-sheet rich structural features of the MspA template from which MAD1 was designed.

Plotting the pH-dependent elliptical change resulting from MAD1's $\beta$-sheet $\left(\theta_{212 n m}\right)$ and tryptophan-zipper $\left(\theta_{228 n m}\right)$ structures demonstrates that the peptide's supramolecular conformational equilibrium shifts at $\mathrm{pH}<6.0$ (Fig. 1h). This aligns with the pKa of histidine's imidazole side chain and suggests these residues play an important role in directing MAD1 fibrilization in the acidic macrophage phagolysosome. Yet, given the polycationic nature of MAD1 it is unusual that its self-assembly would be driven by acidic conditions, rather than disrupted due to intermolecular electrostatic repulsion. In nature, pathway driven self-assembly is a composite of noncovalent forces and solvation effects that direct divergent intermolecular interactions, leading to the potential emergence of nonequilibrium states. ${ }^{17,18}$ To investigate the thermodynamic stability of MAD1 nanostructures, en route to understanding the mechanistic basis for its potential nonequilibrium assembly behavior, we performed thermal change CD studies. MAD1 displays a reversible thermal denaturation curve $\left(T_{m}=39^{\circ} \mathrm{C}\right)$ with forward and reverse melting profiles that are closely overlaid (Fig. 1i, Supplementary Fig. 2). This suggests that MAD1's tryptophan-zippered fibrillar state represents a free energy well, an assertion we next rigorously test through in silico experiments.

\section{MAD1 conformational dynamics}


a

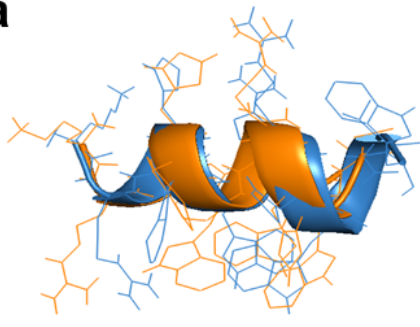

C

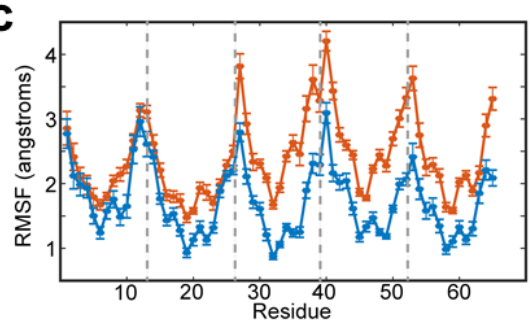

e

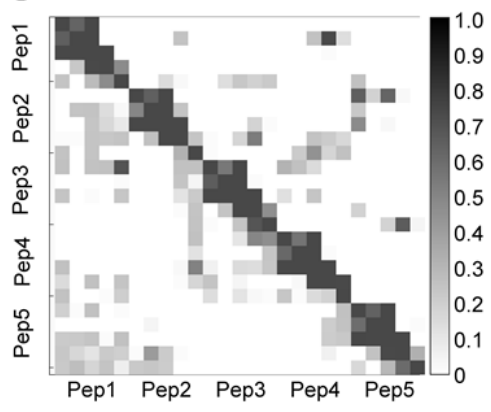

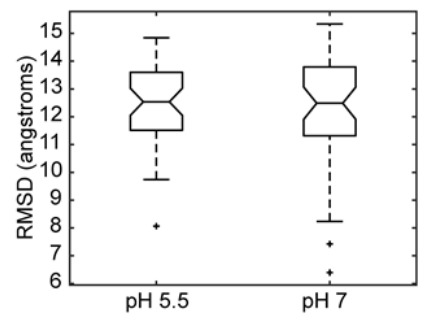
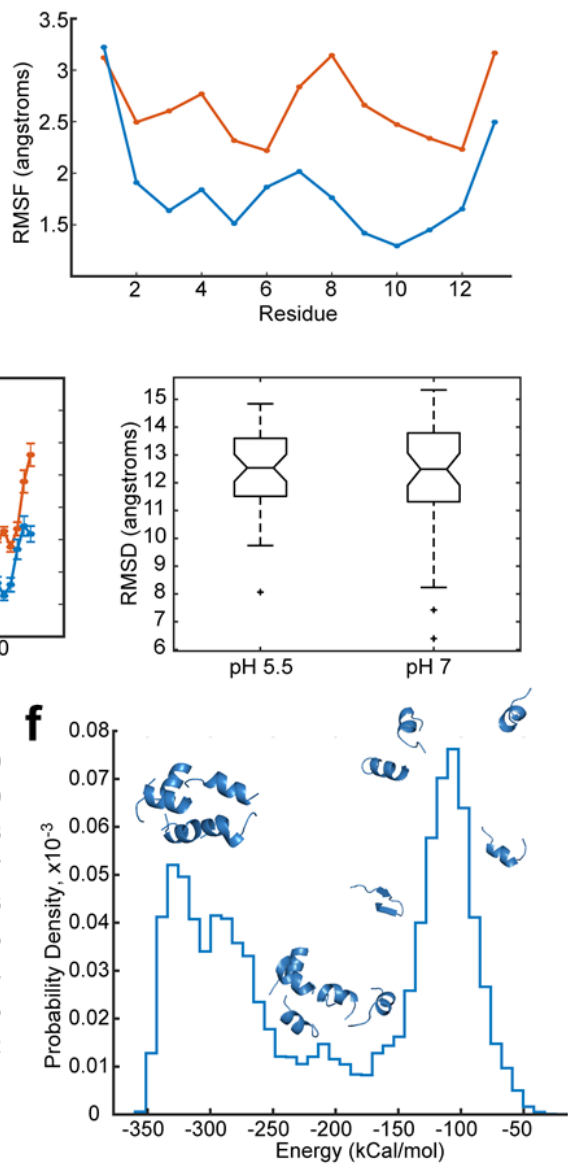

b

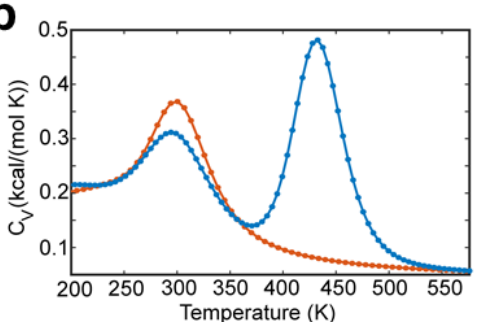

d
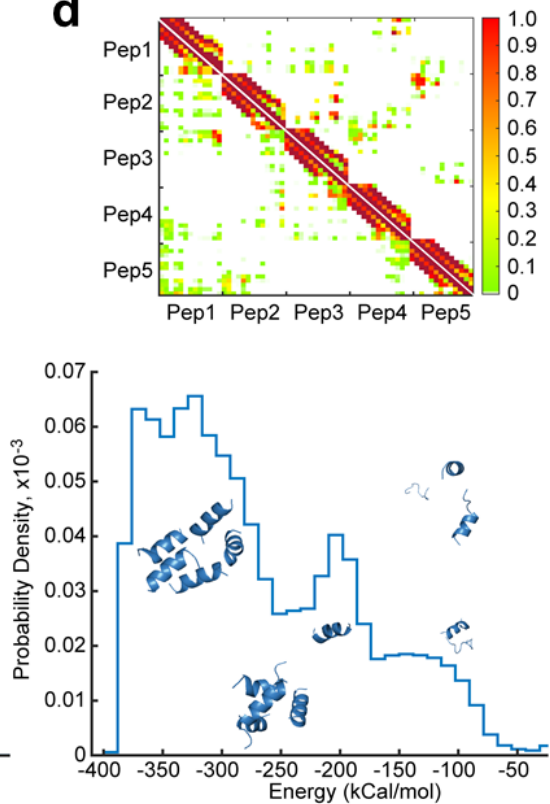

Fig. 2 | Atomistic mechanisms of MAD1 assembly. a, Structure (left) of the MAD1 peptide at pH 7 (blue) and pH 5.5 (orange), and profile of MAD1 dynamics as root mean square fluctuations at each residue over simulation at equilibrium (right). b, Volume-constant specific heat of MAD1 monomer at pH 7 (blue) and pH 5.5 (orange). c, Root mean square fluctuations (left) at each residue of MAD1 pentameric assembly, mean over 10 independent simulations. Vertical dashed lines separate individual monomers, error bars are standard error. As expected, we note peaks of increased fluctuation at peptide $\mathrm{N}$ - and $\mathrm{C}$-termini. Box-whisker plot (right) of pairwise root mean square deviation comparison between consensus structures of 10 randomized simulation iterations of MAD1 pentameric assembly (45 comparisons total for each $\mathrm{pH}$ ). d, Heat map of residue-residue contact frequencies in MAD1 pentamer assembly at $\mathrm{pH} 7$ (upper diagonal) and $\mathrm{pH} 5.5$ (lower diagonal). e, Heat map of tryptophan-tryptophan contact frequencies in MAD1 pentamer assembly at pH 7 (upper diagonal) and pH 5.5 (lower diagonal). f, Normalized histogram of potential energies of structures occurring at energetic transition at pH 5.5 (left) and $\mathrm{pH} 7$ (right). Peaks represent discrete energetic transition states, with representative structures of each energetic state pictured over each peak.

To elucidate the atomistic mechanisms underlying MAD1 self-assembly we performed discrete molecular dynamics (DMD) simulations of the peptide in the unprotonated $(\mathrm{pH} 7)$ and protonated $(\mathrm{pH} 5.5)$ histidine state. In agreement with experiments, we find the peptide monomer forms an alpha-helical structure (Fig. 2a), with similar backbone but differing side chain orientation at the two pHs. Under acidic conditions, MAD1 is highly dynamic, exhibiting significantly increased root mean square fluctuations (RMSF) over the length of the peptide. Although the static structure of MAD1 is similar at acidic and neutral $\mathrm{pH}$, these heightened dynamics suggest decreased thermodynamic stability at $\mathrm{pH}$ 5.5. Using replica exchange simulations, we observe that the melting curve of MAD1 at acidic $\mathrm{pH}$ shifts to the left (Fig. $\mathbf{2 b}$ ), confirming decreased stability when the peptide's histidine side chains are protonated. This decreased thermodynamic stability may increase propensity for aggregation, possibly explaining the fibrils observed in our TEM experiments (Fig. 1g). To test this propensity in silico, we 
performed simulations of five peptides in solution at both acidic and neutral $\mathrm{pH}$. We found that not only are the individual peptides more dynamic even when assembled in a pentamer (Fig. 2c, left), but that assembly is more chaotic and the structural space of potential pentamers is more highly degenerate, as evidenced by comparison of structures resulting from ten randomized simulation iterations (Fig. 2c, right). Further, we found that this structural plasticity in MAD1 pentamers exhibited more frequent residue-residue contacts at acidic pH (Fig. 2d, bottom diagonal). The structural plasticity was also reflected across tryptophan-tryptophan residue contacts at acidic $\mathrm{pH}$ (Fig $\mathbf{2 e}$, bottom diagonal). Increased frequency of tryptophantryptophan interactions at low $\mathrm{pH}$ reflects promoted formation of a tryptophan zipper motif (Fig. 2e, bottom diagonal). In contrast, we observe fewer, but stronger, interactions at neutral $\mathrm{pH}$, suggesting a less diverse ensemble of structural states (Fig $\mathbf{2 d} \boldsymbol{\&} \mathbf{e}$, top diagonals). To further explore these atomistic mechanisms of peptide assembly, we examined structural states of MAD1 at energetic transitions (Fig. 2f). Similar energetic states were identified of approximately -330 kcal/mol (fully assembled pentamer), -205 kcal/mol (partially assembled pentamer), and -125 $(\mathrm{pH} \mathrm{7)}$ or $-105(\mathrm{pH} 5.5) \mathrm{kcal} / \mathrm{mol}$ (dissociated monomers maintaining at least partial secondary structure). The shift toward higher energy in this dissociated structure denotes decreased stability of separated monomers at $\mathrm{pH}$ 5.5. Together, this demonstrates that increased conformational flexibility of MAD1 at acidic $\mathrm{pH}$, caused by intramolecular electrostatic repulsion, favors more dynamic intermolecular interactions relative to neutral conditions, which in turn confers a higher energetic benefit to assembly and a greater barrier to dissociation once oligomers have formed. This has pathophysiologic significance as the $\mathrm{pH}$ of the macrophage phagolysosome, where Mtb reside during early infection, evolves from $\mathrm{pH} 6.2$ to 4.5 after microbial ingestion. ${ }^{11,19}$ This suggests that triggered assembly of MAD1 in the acidic phagolysosome may provide a critical defense against TB pathogens that exploit this host niche for survival.

\section{Antitubercular specificity conferred via myco-favored interactions}

Bactericidal specificity of MAD1 was next tested against a diverse microbiologic panel containing mycobacteria, as well as a variety of Gram-negative and Gram-positive lung commensals and opportunistic respiratory pathogens. Results in Table 1 demonstrate that MAD1 elicits potent activity towards its Mtb target, with a minimum inhibitory concentration (MIC) similar to many first-line TB antibiotics (MICs $\sim 0.5-20 \mu \mathrm{M}$ ). ${ }^{20}$ In addition, MAD1 is active towards Mycobacterium smegmatis (M. smegmatis), a non-pathogenic mycobacteria that is also defined by abundant MspA content in its cellular envelope. Importantly, the chiral enantiomer of MAD1, prepared from non-natural $D$-amino acids (Supplementary Fig. 3), is equally as potent as its natural $L$-analogue towards both of the tested mycobacteria. This suggests that MAD1's antimycobacterial activity occurs through a physical mechanism, presumably by disrupting the mycolic acid-rich cell envelope, as opposed to a chirality-sensitive biomolecular interaction. 
Remarkably, MAD1 displays an exceptional myco-specificity relative to other Gram-negative and Gram-positive respiratory commensals, with a selectivity index (SI) of 20 . This specificity is an order of magnitude greater than three other tryptophan-rich, antimycobacterial peptides tested, which were selected based on their reported anti-TB activity (AMPs1-3 in Table 1). ${ }^{21}$ MAD1 is similarly discriminatory when compared against many TB antibiotics, which generally have Sl's $\leq 2 .^{20}$ These results suggest that MAD1 may elicit its antimicrobial activity through a membrane composition-dependent mechanism, thus enabling it to distinguish mycobacteria from Gram-positive and Gram-negative counterparts.

Table 1: AMP minimum inhibitory concentration (MIC)

\begin{tabular}{|c|c|c|c|c|}
\hline & \multicolumn{4}{|c|}{ Peptide MIC $[\mu \mathrm{M}]$} \\
\hline & $\begin{array}{l}\text { MAD1 } \\
\text { L(D) }\end{array}$ & AMP1 & AMP2 & AMP3 \\
\hline \multicolumn{5}{|l|}{ Mycobacteria } \\
\hline M. tuberculosis & $2.5(2.5)$ & 20 & 10 & 20 \\
\hline M. smegmatis & $5(5)$ & 20 & 20 & 20 \\
\hline \multicolumn{5}{|l|}{ Gram-Positive } \\
\hline MR S. aureus ${ }^{b}$ & $>80$ & $>80$ & 80 & 20 \\
\hline MS S. aureus ${ }^{b}$ & $>80$ & $>80$ & 80 & 20 \\
\hline B. anthracis & $>80$ & 10 & 80 & 20 \\
\hline \multicolumn{5}{|l|}{ Gram-Negative } \\
\hline K. pneumoniae & $>80$ & 20 & 20 & 2.5 \\
\hline$P$. aeruginosa & 50 & 10 & 20 & 80 \\
\hline A. baumannii & 50 & 25 & 80 & 10 \\
\hline H. influenzae & 80 & 80 & 80 & 40 \\
\hline Selectivity Inde) & 20 & $<1$ & 2 & $<1$ \\
\hline
\end{tabular}

Peptide sequences (prepared with amidated $C$-terminus): MAD1: KRWHWWRRHWVVW-NH ${ }_{2}$; AMP1: WKWLKKWIK-NH AMP2: KRWWKWWRR-NH $\mathrm{N}_{2}$; AMP3: RRWWRWVVW- $\mathrm{NH}_{2}$

aL-peptide ( $\mathrm{D}$ - chiral enantiomer); ${ }^{\mathrm{b}} \mathrm{MR}=$ Methicillin-resistant; MS $=$ Methicillin-sensitive; 'Selectivity index calculated as $\mathrm{MIC}_{\text {lowest }} / \mathrm{MIC}_{\mathrm{Mtb}}$. 

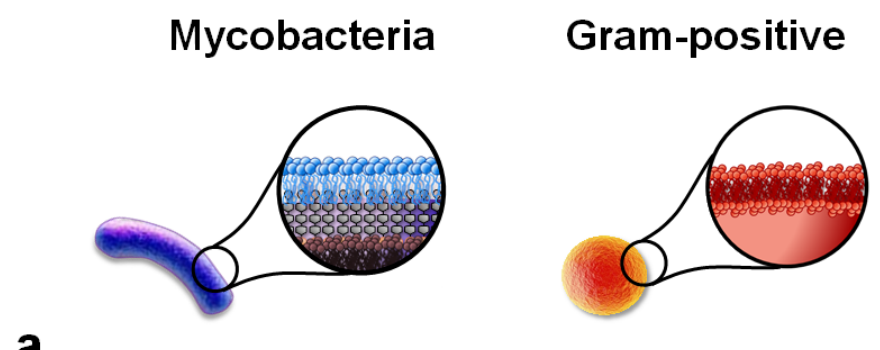

\section{Gram-negative}

a
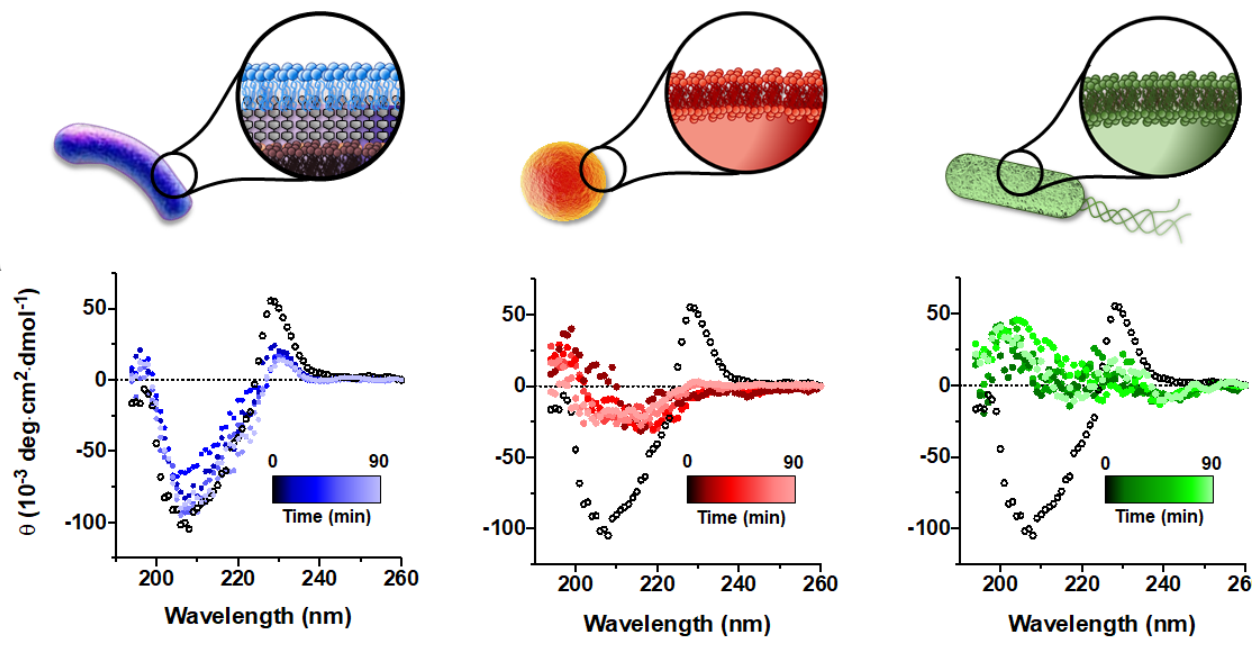

b
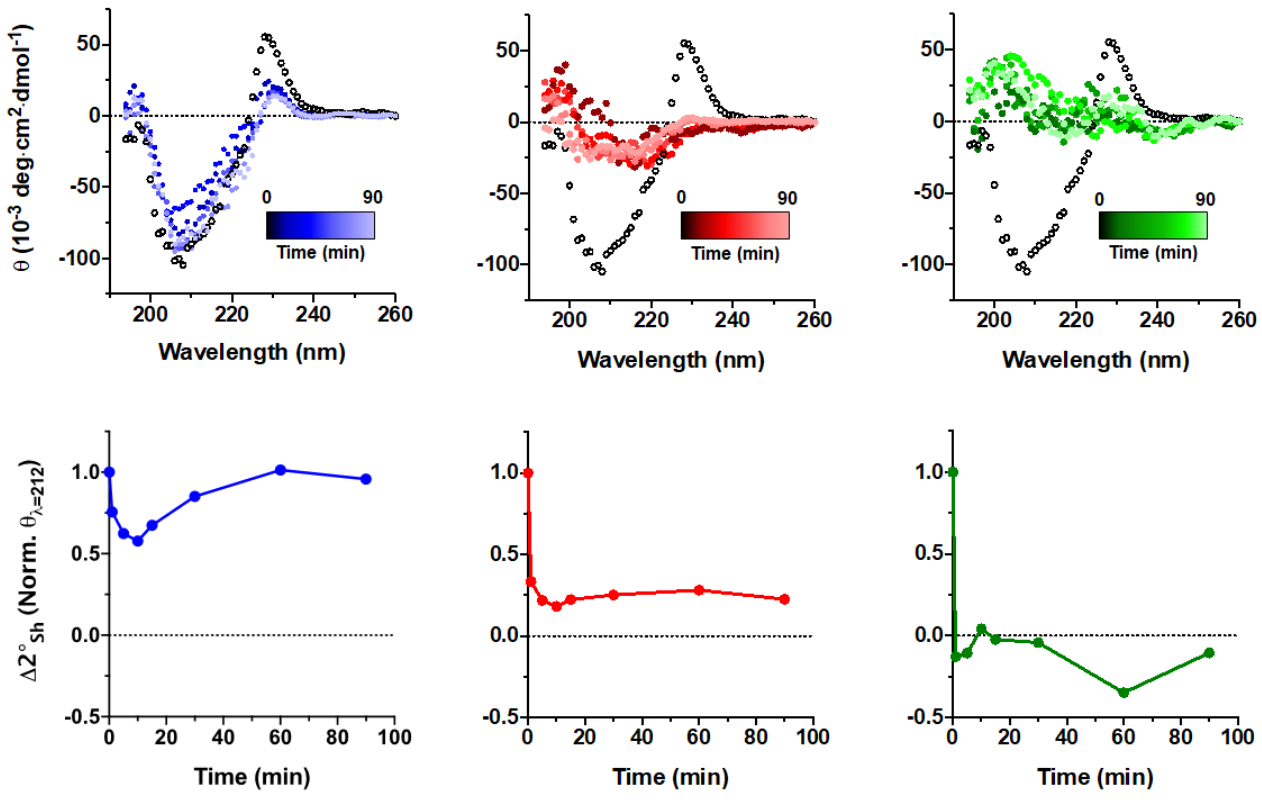

C
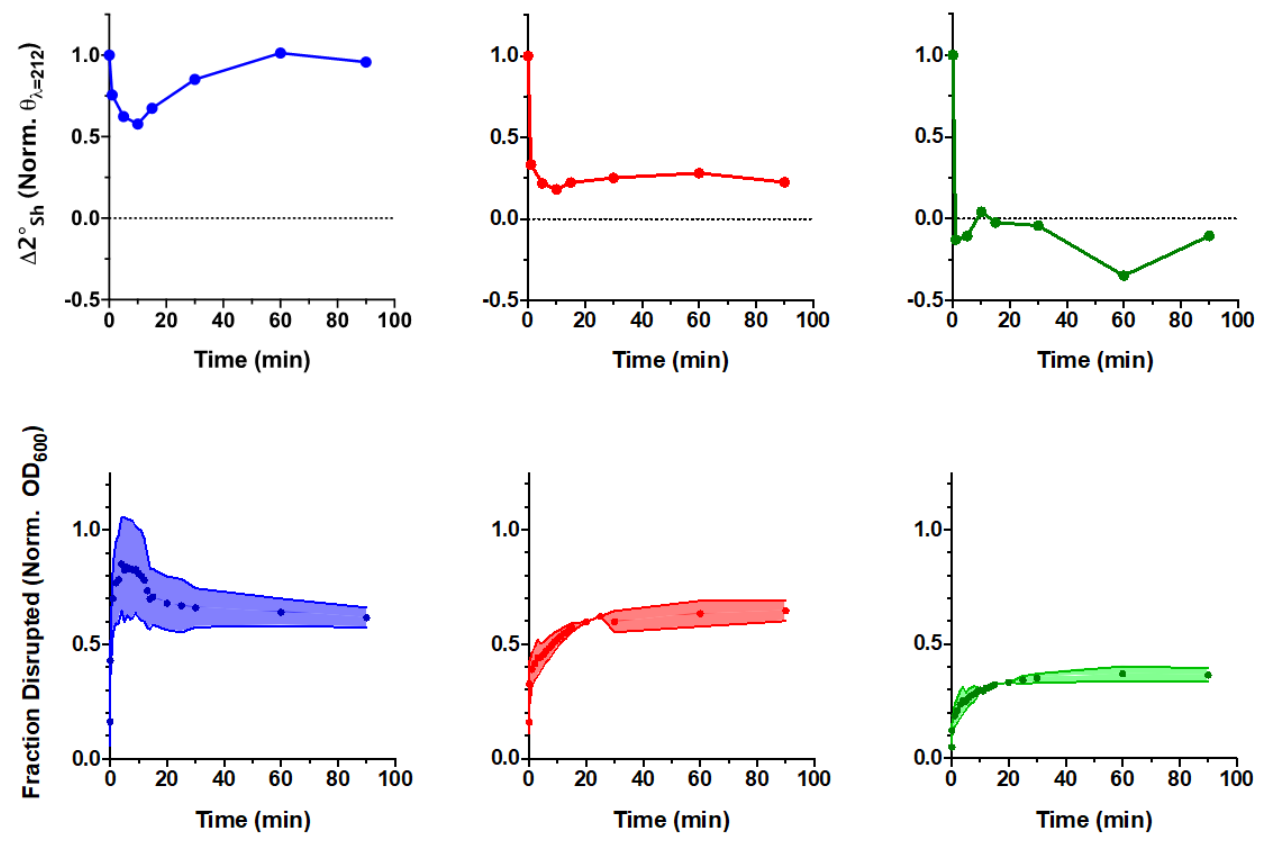

Fig. 3 | Ex cellulo analysis of MAD1 myco-membrane specificity. a, Circular dichroism spectrum of MAD1 in the presence of model mycobacterial (left, blue), gram-positive (red, middle) and gram-negative (green, right) liposomal membrane analogues. Peptide spectra at $t=0$ represented in open black circles, and $t \rightarrow 90$ min. shown as increasing lighter shades of color. $\mathbf{b}$, Magnitude change in MAD1 $\beta$-sheet secondary structure $\left(\Delta 2^{\circ}\right.$ sh) over time, as derived from CD spectra shown in panel a. Dotted line demarcates complete loss of sheet-rich architectures. c, Fractional disruption of model microbial membranes determined from optical density measurements (OD ${ }_{600}$ ). Measurements normalized to unextruded lipid dispersions as a positive lytic control. Symbols represent mean value; shaded regions report SEM. $\mathrm{n} \geq 3$ for all experiments, with representative CD spectra shown.

To test this assertion, we next investigated the conformational dynamics of MAD1 in the presence of liposomal myco-membrane models. CD spectra presented in Fig. 3a shows a slight, transient loss of MAD1's helical and $\beta$-sheet conformational states $\left(\theta_{200-220 \mathrm{~nm}}\right)$ upon interaction with membranes prepared from the Mtb cell envelope glycolipid, trehalose dimycolate. Plotting the magnitude change in MAD1 $\beta$-sheet structure $\left(\theta_{212 n m}\right)$ reveals this transition peaks at 10 minutes, and then reverses to restore its initial sheet-rich architecture 
(Fig. 3b). Conversely, MAD1 is rapidly denatured upon mixing with both the Gram-positive and Gram-negative model membranes, and is unable to recover its secondary structure over the remainder of the experiment (Fig. $3 a$ and b). In separate studies, optical density measurements were used to monitor the disruption of each bacterial membrane in the presence of elevated MAD1 concentrations. Results in Fig. 3c demonstrate that nearly $100 \%$ of myco-membranes are lysed within ten minutes following the addition of the peptide. Interestingly, this rapid lytic action matches the kinetics of MAD1's conformational states upon myco-membrane insertion (Fig. 3b), suggesting a direct link between peptide structure and myco-lytic potential. Under similar conditions MAD1 elicits modest disruption of Gram-positive and Gram-negative liposomes ( $\sim 65 \%$ and $\sim 35 \%$, respectively) and is weakly active towards mammalian control membranes prepared from lipid compositions matching healthy lung epithelium (Supplementary Fig. 4). Taken together, these results provide strong evidence for the selective potentiation of MAD1's lytic action at the surface of myco-membranes and demonstrates its potential to precisely kill Mtb pathogens in polymicrobial commensal environments without damage to healthy host tissue; an assertion we validate in later studies.

Although our results clearly demonstrate myco-specific action of the peptide, the modest disruption of Gram-positive membranes by MAD1 appears to contradict its near complete inactivity towards Gram-positive microbes in our bacteriologic assays (Table 1). These divergent results may be a consequence of the lack of a peptidoglycan layer in our model liposomes. This is important because, while both organisms share a similar inner membrane composition, Grampositive microbes have a multi-layered peptidoglycan coating that is generally thicker than that of mycobacteria. ${ }^{22,23}$ This dense glycan interface may preferentially adsorb MAD1 and prevent its ability to partition to the inner membrane, thus insulating Gram-positive lung commensals from peptide-mediated lysis. Supporting this assertion, plasmon resonance (SPR) experiments demonstrate that MAD1 avidly binds peptidoglycans isolated from the Gram-positive commensal S. aureus ( $\mathrm{K}_{\mathrm{D}}=8 \mu \mathrm{M}$; Supplementary Fig. 5).

\section{Mtb-instructed MAD1 assembly into supramolecular nano-lytics}

Thus far, our ex cellulo experiments demonstrate that MAD1's lytic activity is conferred via its preferential interactions with mycolic acids. We next explore if these same membranetemplated lytic phenomena occur in the context of living Mtb cells, first through a fluorometric permeabilization assay. Here, the Mtb outer mycolic-acid surface is selectively labeled using the membrane potential sensitive dye, 1-N-phenylnaphthylamine (NPN). In Gram-positive and Gram-negative bacteria this impermeant dye is excluded from intact membranes, but enters damaged phospholipid layers to display prominent fluorescence. ${ }^{24-27}$ Interestingly, we observed an inverse behavior of NPN in Mtb cells. The probe readily intercalates into the hydrophobic outer myco-membrane of healthy Mtb cells to generate a strong fluorescent signal (Supplementary Fig. 6). This unique activity enables us to use NPN fluorescence to directly measure changes in Mtb outer membrane stability. Results in Fig. 4a show a dose-dependent loss of Mtb outer membrane integrity upon addition of MAD1, suggesting either disruption or removal of the hydrophobic mycolic coating by the peptide. Conversely, incubating NPN-labeled Mtb with a lytic concentration of digitonin, a non-ionic peptide detergent used to permeabilize bacterial membranes, did not result in a significant decrease in fluorescence. Taken together, this in vitro evidence provides further support for a myco-membrane selective mode of action for MAD1 that is not shared by promiscuous broad-spectrum bacteriolytics (e.g. digitonin), and by extension unlikely employed by the naturally derived tryptophan-rich antimicrobial peptides we previously tested (Table 1). 
a

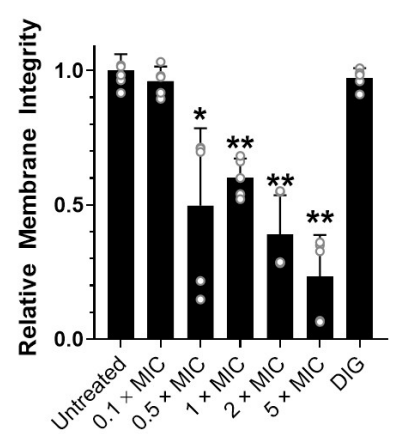

d

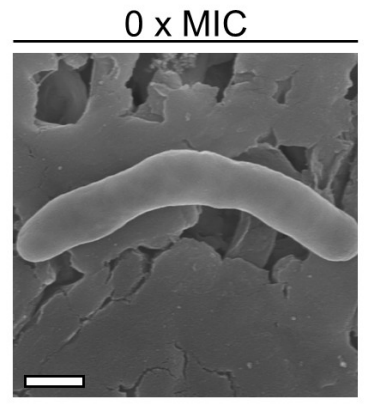

e

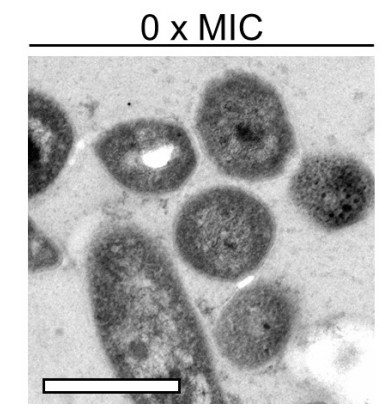

b
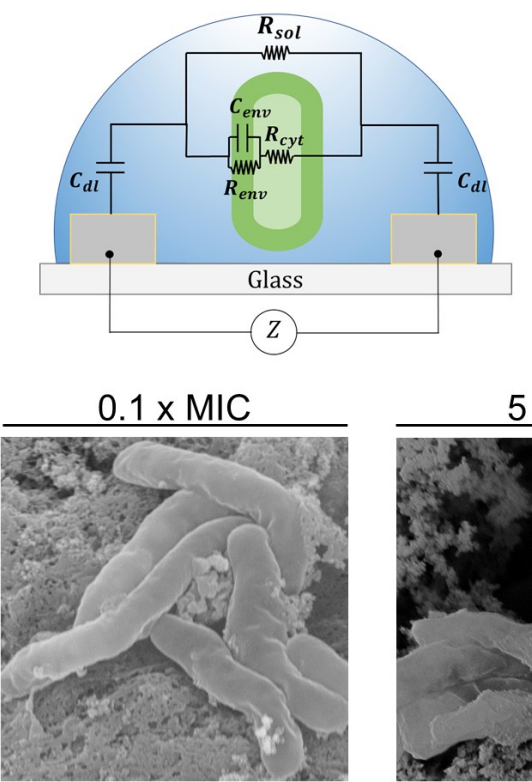

$0.1 \times$ MIC

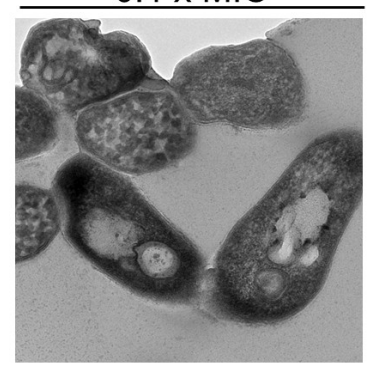

C

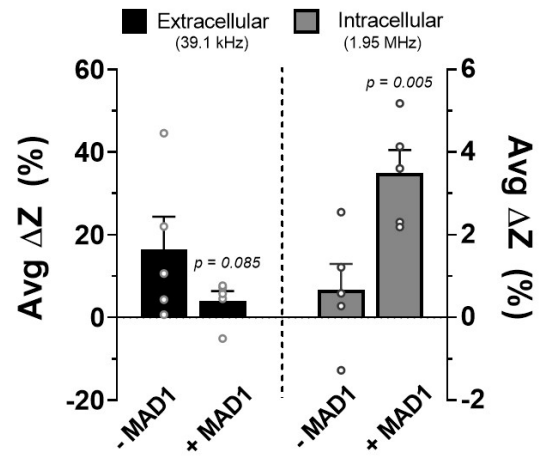

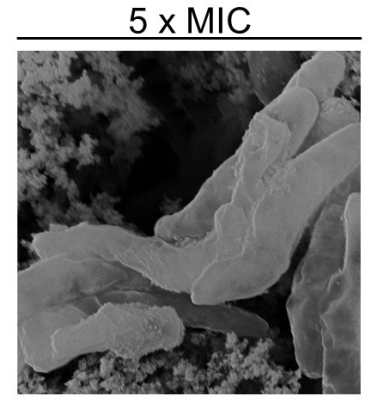

$5 \times \mathrm{MIC}$

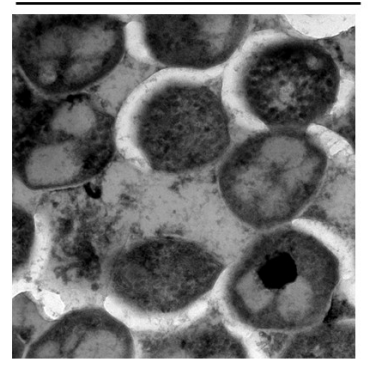

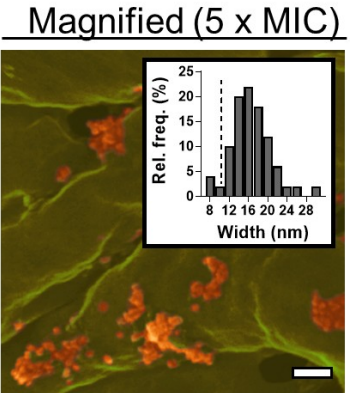

Magnified (5 x MIC)

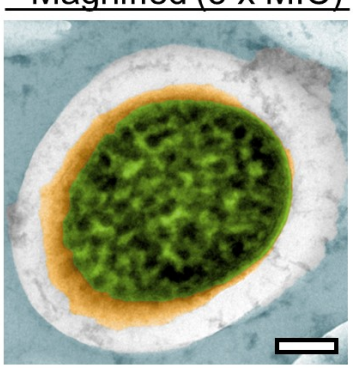

Fig. 4 | In situ characterization of Mtb envelope disruption by MAD1. a, Relative membrane integrity of Mtb in the absence (untreated) or presence $(x$ MIC) of increasing MAD1 concentrations (DIG = digitonin). Individual data points shown as open circles $(n=5)$, and bars represent mean \pm S.D. Statistical significance determined using a one-tailed Student's t-test relative to untreated control, with * indicating $p<0.01$ and ** indicating $p<0.001$. b, Circuit diagram utilized to model system impedance for IS experiments. Total impedance is comprised of solution resistance

$\left(R_{\text {sol }}\right)$, cell envelope resistance $\left(R_{\text {env }} \dot{b}\right.$, cell envelope capacitance $\left(C_{\text {env }}\right)$, cytoplasmic resistance

$\left(R_{c y t}\right)$, and double-layer capacitance $\left(C_{d l}\right)$. c, Left: Percent change of solution impedance $(\Delta Z @ 39.1$ $\mathrm{kHz}$ ) in the absence (Mtb) or presence (Mtb + MAD1) of MAD1 at $1 \times$ MIC. In the absence of cells, total impedance is dominated by $R_{\text {sol }}$ and $C_{d l}$. Right: Percent change in cell envelope impedance $(\Delta Z @ 1.95 \mathrm{MHz})$ in the absence or presence of MAD1 at 1 x MIC. Processed impedance spectra, and associated raw data, are shown in Supplementary Figs. 7 and 8, respectively. Individual data points shown as open circles $(n=5)$, and bars represent mean \pm S.E.M. Statistical significance determined relative to untreated control, with $p$ values reported from a onetailed Student's t-test. d, SEM and e, TEM micrographs of Mtb cells before $(0 \times \mathrm{MIC})$ and after treatment with MAD1 at $0.1 \mathrm{x}$ or $5 \mathrm{x}$ MIC. Scale bar $=0.5 \mu \mathrm{m}$. Right: Magnified and false-colored EM images of the Mtb (d) outer surface (green = Mtb cell envelope, red = MAD1 supramolecular structures) and (e) cellular cross-section following treatment with 5 x MIC MAD1 (green = Mtb cytoplasm, yellow = intracellular leakage, white space = delaminated cell wall). Panel $d$ inset: histoqram of MAD1 cvlindrical assembly width (n = 50) : dashed line shows the reported diameter of the To corroborate these results, we next investigated intb membrane dynamics in the presence of MAD1 through a series of label-free impedance spectroscopy (IS) experiments. This technique measures the current between two electrodes induced by an alternating voltage to calculate a complex impedance, $Z(\omega)$, where $\omega$ is the voltage frequency. ${ }^{28,29}$ When applied to bacterial cells, frequency-dependent changes in impedance can be used to infer 
properties of the extracellular and intracellular milieu upon treatment with MAD1. To facilitate interpretation of this complex system we use a generalized biologic circuit (Fig. 4b) and designed two complimentary experiments to study MAD1-mediated changes in Mtb membrane integrity. In the first study (Fig. 4c, black bars), we employed low frequency electric fields to measure changes in supernatant impedance ( $\Delta Z$ ) upon treatment of Mtb with MAD1, which can indicate cytoplasmic leakage into the extracellular space. As expected, we observed a decrease in the extracellular solution impedance (equivalently the resistance) as intracellular ions escape the MAD1-permeabilized mycobacterial envelope (processed impedance spectra, and associated raw data, are shown in Supplementary Figs. 7 and 8, respectively). In the second experiment (Fig. 4c, grey bars), a high frequency electric field is employed that, in contrast to low frequencies, penetrates the mycobacterial envelope to directly measure cytoplasmic impedance. Results show that $\Delta \mathrm{Z}$ of the Mtb cellular compartment increases following treatment with the peptide, indicating a loss of intracellular ionic content (i.e. an increase of cytoplasmic resistance) due to cell envelope damage. Recent dielectrophoresis studies have similarly shown that mycobacterial cell envelope capacitance and cytoplasm conductivity decrease (hence, $\Delta \mathrm{Z}$ increases) upon treatment with heat or the antibiotic isoniazid $(\mathrm{INH})$, both of which induce morphological cell wall changes. ${ }^{30}$ These previously reported results, together with our findings, strongly support a membrane-directed antitubercular mechanism of action for MAD1 that compromises Mtb envelope integrity, causes leakage of the cytoplasmic contents and elicits rapid mycobactericidal activity.

In separate studies, we utilized scanning and transmission electron microscopy to directly probe MAD1-mediated morphologic changes in the Mtb envelope architecture. In the presence of sub-lytic concentrations of the peptide $(0.1 \times \mathrm{MIC})$ the typically smooth Mtb surface begins to display a ruffled morphology that is complimented by the formation of extracellular aggregates (Fig. 4d). Increasing the concentration of MAD1 to $0.5 x-5 x$ its MIC led to a stark change in cell surface morphology characterized by the formation of pitted architectures and delamination of the cell wall (Fig. 4d and Supplementary Fig. 9). Remarkably, close inspection of the Mtb cell surface reveals the formation of MAD1 cylindrical assemblies that are $\sim 14 \mathrm{~nm}$ in diameter (Figure $4 \mathrm{~d}$, magnified image and inset), which approximates the size of the MspA porin head (9 $\mathrm{nm}$ in width). ${ }^{31}$ TEM images shown in Fig. 4e demonstrate that at low MAD1 concentrations (0.1 $x$ MIC) cytoplasmic granules begin to develop, which are indicative of an early cellular stress response. At elevated peptide concentrations $(0.5 x-5 \times \mathrm{MIC})$ a low density corona is observed circumscribing the treated Mtb cell (Fig. 4e and Supplementary Fig. 10, white space around cell). This electron-deficient outer halo is most likely formed due to MAD1-mediated exfoliation of the outer mycobacterial membrane. Additionally, we observed a significant amount of cellular debris forming in the extracellular space when treated at high peptide concentrations. This further corroborates the impedance spectroscopy and SEM imaging, and strongly supports our assertion of MAD1-mediated membrane poration that ultimately leads to Mtb cell death via cytoplasmic leakage.

\section{MAD1 polymicrobial specificity and antibiotic synergy}

Next, we tested the ability of MAD1 to selectively engage its mycobacterial target in a complex polymicrobial milieu. In our initial experiments, Mtb cells are mixed with the Grampositive and Gram-negative commensals S. aureus and $K$. pneumoniae, respectively, before addition of the MAD1 peptide. Treated cultures are then subsequently labeled with auraminerhodamine, a fluorescent dye that selectively accumulates in acid-fast mycobacteria. ${ }^{32}$ Like NPN, this dye enables specific interrogation of Mtb cell integrity in the mixed commensal population. Results in Fig. 5a show a significant decline in Mtb integrity as MAD1 concentration is increased from 1.0x - 10 x MIC. In parallel experiments, Mtb-specific killing was tested by comparing the growth rate of Mtb against co-treated commensals in the presence of MAD1. To select an appropriate peptide dose, cytotoxicity curves were generated for each species as a 

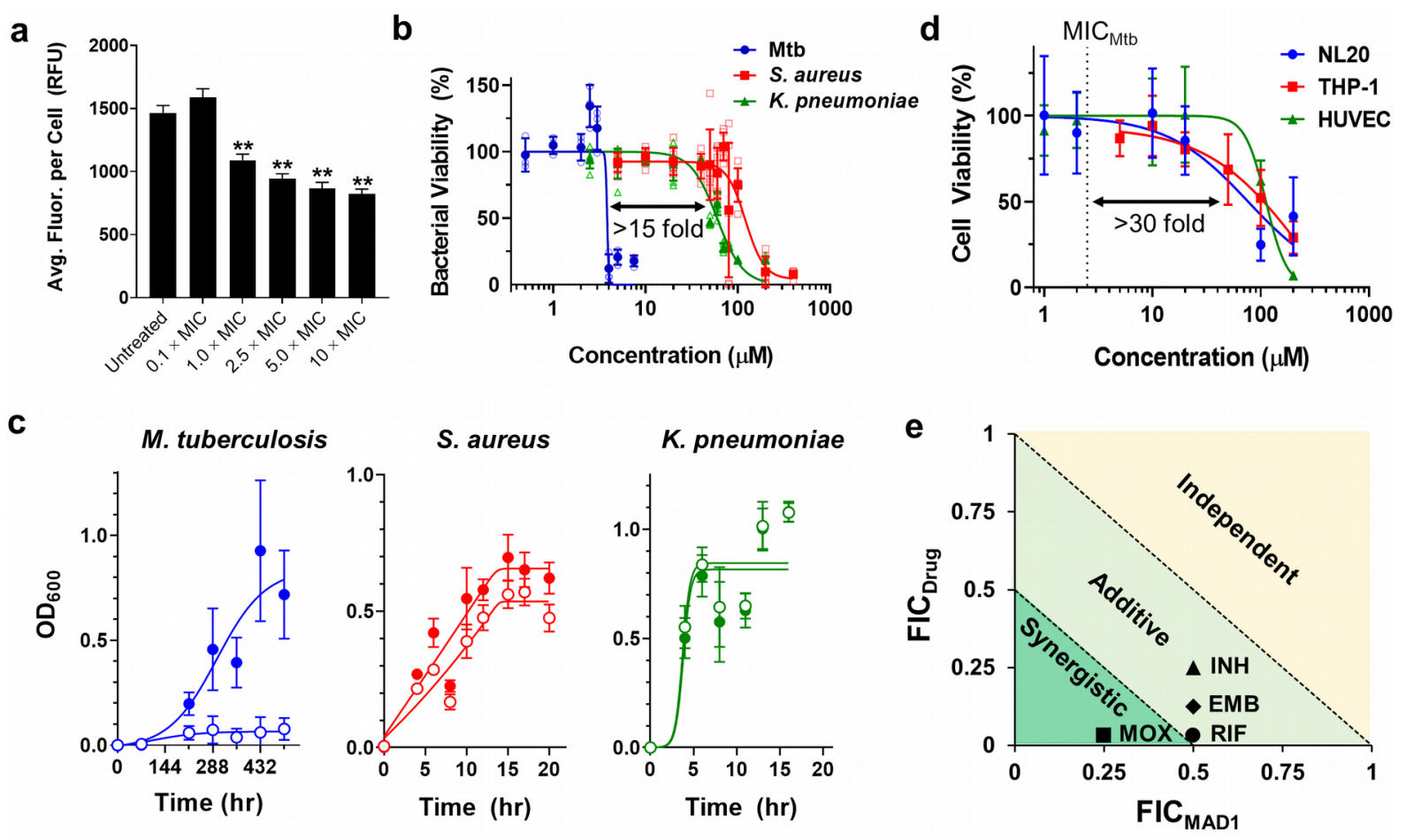

Fig. 5 | MAD1 polymicrobial selectivity and combinatorial synergy. a, Relative membrane integrity of Mtb cells in the absence (untreated) or presence (x MIC) of increasing MAD1 concentrations. Membrane disruption quantified by auramine-rhodamine staining in a polymicrobial co-culture of Mtb with $S$. aureus and $K$. pneumoniae. Bars represent mean \pm S.E.M. and statistical significance determined via a two-tailed Student's t-test relative to untreated control, with ** indicating $p<0.001$. b. Bacterial viability of $M t b$, or the model commensals $S$. aureus and $K$. pneumoniae, following treatment with increasing concentrations of MAD1. Individual data points (open symbols) are shown behind mean values \pm S.D. $(n \geq 3)$. c, Polymicrobial growth curves monitoring the proliferation of each bacterial strain in the absence $(\bullet)$ or presence $(0)$ of MAD1 at $4 \times \mathrm{MIC}_{\mathrm{Mtb}}$, shown as mean \pm S.D. d, Cytotoxicity of MAD1 against pathophysiologically relevant mammalian cell lines, including human normal lung epithelium (NL20), monocytederived macrophages (THP-1) and vascular endothelial cells (HUVEC), shown as mean \pm S.D. e, Combinatorial synergy of MAD1 with first-line TB antibiotics isoniazid (INH), ethambutol (EMB) and rifampicin (RIF), as well as the second line anti-tubercular drug moxifloxacin (MOX). Fractional inhibition concentration (FIC) $<1$ and $<0.5$ are considered additive and synergistic, respectively. Data is presented as the FIC contribution of MAD1 against the contribution from the small molecule antibiotic.

function of increasing MAD1 concentration (Fig. 5b). These curves identified a therapeutic window for MAD1 of $2-20 \mu \mathrm{M}$, between which complete killing of Mtb is achieved without significant toxicity towards the commensals $S$. aureus and K. pneumoniae. Choosing a MAD1 concentration within this range, $10 \mu \mathrm{M}\left(4 \times \mathrm{MIC}_{\mathrm{Mtb}}\right)$, we next treated each strain with the peptide and monitored their time-dependent growth. Results in Fig. 5c show that the proliferation rate of $S$. aureus or $K$. pneumoniae commensals was not changed upon addition of the peptide. Conversely, Mtb growth was completely inhibited in culture by the MAD1 peptide.

In separate studies, MAD1 was found to be well tolerated by a panel of human cell lines with pathophysiologic relevance to pulmonary TB, including NL-20 (lung epithelium), THP-1 (monocyte-derived macrophages) and vascular endothelial cells (HUVEC). All three cell lines remained viable when treated with concentrations of MAD1 well above its $\mathrm{MIC}_{\mathrm{Mtb}}$ (see dashed line in Fig. 5d), resulting in therapeutic indices of $30-43$ (Supplementary Table 1). Taken together with results from our bacteriologic assays, this data strongly suggests that MAD1 maintains its myco-membrane targeted mechanism of action in complex commensal mixtures, leading to precise anti-TB activity without collateral toxicity of lung microflora or host tissue. 
Given MAD1's ability to selectively permeabilize the rigid outer mycobacterial membrane, we employed checkboard assays to assess the peptide's potential to synergistically enhance the efficacy of select first- and second-line TB antibiotics. This has important clinical implications as poor intracellular permeation of many TB drugs necessitates high doses and long treatment durations to achieve successful clinical outcomes. As shown in Fig. 5e, MAD1 displays additive effects with first-line agents isoniazid (INH), ethambutol (EMB) and rifampicin (RIF), and potently synergizes with the second-line agent moxifloxacin (MOX). Interestingly, the Niederweis group has shown that the MspA porin facilitates diffusion of MOX into mycobacteria and contributes to its antitubercular effects. ${ }^{33}$ Correspondingly, $M$. smegmatis deletion mutants that lack the ability to express Msp porins have a pronounced resistance to MOX compared to the wild-type strain. This suggests that MAD1 may synergistically enhance the activity of MOX by forming inoperative, MspA-mimetic pores in the Mtb envelope that further potentiate the intrabacilli transport of the antibiotic.

\section{Discussion}

We have established a biomimetic design strategy that can be used to directly prototype de novo, pathogen-selective host defense peptides. This approach eliminates the need for large empirical screens and, hence, allows for a robust and rapid antimicrobial discovery pipeline. A unique advantage of this methodology is that narrow-spectrum peptides may be produced atscale using insensitive bacterial expression systems (MAD1 for example is inactive towards $E$. coli); a production method that is otherwise inaccessible to naturally-derived broad-spectrum antimicrobials. More importantly, this strategy can be used to develop antimicrobials that do not target a particular biochemical pathway, but instead act by exploiting physical structural features of the cell envelope that are difficult for pathogens to mutationally alter.

As an exemplary application we have prepared a first-in-class, TB-specific host defense peptide, MAD1, inspired by the mycobacterial-specific porin MspA. MAD1 organizes into tryptophan-zippered supramolecular assemblies that recapitulate the $\beta$-sheet rich architecture of the porin template from which its derived. We show that this biomimetic behavior confers MAD1 with the ability to selectively target the mycolic-acid rich surface of Mtb to elicit rapid and precise anti-TB activity. Importantly, because MAD1 utilizes a physical mechanism of action, we are not limited to natural $L$-amino acids and can instead employ protease-resistant non-natural $D$ enantiomeric sequences. Further, these membrane active mechanisms potentiate the activity of other drugs against Mtb by permeabilizing the rigid myco-envelope that otherwise inhibits the intracellular partitioning of antibiotics, and can specifically aid the porin-mediated diffusion of fluoroquinolones into mycobacteria.

Although applied here to TB, there are numerous pathogens with varied and unique channel-forming membrane proteins with which to rationally design biomimetic antimicrobials. Therefore, this work is poised to open a new, diverse area of precision antimicrobials capable of combating MDR respiratory infections without impacting lung commensals and host tissue.

\section{Acknowledgements}

We acknowledge and thank the Penn State Microscopy and Cytometry Facility - University Park, PA for assistance with confocal and electron microscopy. We also acknowledge the Penn State X-Ray Crystallography Facility - University Park, PA for use of the CD spectrophotometer. Funding for this research was provided by the Penn State Institute of Energy and the Environment Human Health and the Environment Seed Grant awarded to S.H.M. A. W. S was supported by funds from the Penn State Graduate Research Fellowship.

\section{Author Contributions}

A.W.S. and S.H.M. conceived the hypothesis, designed the experiments, and wrote the manuscript. A.W.S., M.R.A., A.S.M., D.C.C., A.B., A.E., E.A.P. and K.C.K designed and 
performed the experiments, analyzed the results and contributed to writing of the manuscript. J.A. contributed to the design of experiments.

\section{Competing interests}

The authors declare no competing interests. 


\section{Methods}

Materials. Lysogeny broth, Mueller Hinton broth, 7H9 media, formic acid, Acetonitrile, auramine/rhodamine, decolorizer, potassium permanganate counterstain, $\mathrm{HCl}$, dichloromethane, 1,2-Ethanedithiol, diethyl 1,3bis(tris(hydroxymethyl)methylamino)propane (BTP), piperadine, spec dmso, thioanisole, sodium fluoride, sodium hydroxide, dimethyl sulfoxide cell culture grade (DMSO), biotech CE dialysis tubing (MWCO 300kD), Gram Stain, DMEM, 96 well tissue culture plates L-glutamine, FBS, trypsin and RPMI media were purchased from ThermoFisher Scientific. Anisole was purchased from Tokyo Chemical Industry. Dimethylformamide, ethanol, F12 media, gentamycin, sodium phosphate monobasic and sodium phosphate dibasic were purchased from VWR. Glycerol, tween 80, OADC growth supplement, BHI, S. aureus peptidoglycans, DiOC2(3), sterile ThermalSeal films, Triton X-100, MEM non-essential amino acid solution, D-(+)-Glucose, Insulin (Recombinant human), Transferrin Apo- (human plasma), EmbryoMax Ultrapure Water with $0.1 \%$ Gelatin, hydrocortisone and sodium bicarbonate were purchased from Millipore-Sigma. Fmoc-protected amino acids, rink amide resin, Oxyma, DIC, 3-(4,5-dimethyl-2-thiazolyl)-2,5diphenyl-2H-tetrazolium bromide (MTT), $\mathrm{N}, \mathrm{N}^{0}$-diisopropylcarbodiimide (DIC), OG surfactant and Trifluoroacetic acid were purchased from Chem-Impex Int. 2-dioleoyl-sn-glycero-3phosphoethanolamine (DOPE), 1,2-dioleoyl-sn-glycero-3-phospho-(1'-rac-glycerol) (DOPG), cardiolipin (CL) and 1-Palmitoyl-2-oleoyl-sn-glycero-3-phosphocholine (POPC) lipids were purchased from Avanti. SPR carboxy-functionalized chips were purchased from Reichert. Trehalose-6,6-dimycolate (TDM) was purchased from InvivoGen.

Cell lines and culture conditions. HUVEC, THP-1, Vascular Cell Basal Medium and the Endothelial Growth Cell Kit-VEGF were purchased from ATCC. NL20 cell line was generously gifted by the laboratory of Matthew Taylor, Penn State University, College of Medicine. NL20 (CRL-2503) cells were cultured in Ham's F12 as recommended by ATCC. HUVEC (PCS-100010) were cultured in supplemented Vascular Cell Basal Medium and grown in flasks coated for 15 min at $37{ }^{\circ} \mathrm{C}$ with $0.1 \%$ gelatin. THP-1 (TIP-202) cells were cultured in RPMI-1640 medium supplemented with 10\% FBS and $50 \mathrm{mM}$ 2-mercaptoethanol (BME). All cultures were maintained at $37{ }^{\circ} \mathrm{C}$ under $5 \% \quad \mathrm{CO}_{2}$ with $0.1 \%$ gentamycin. Bacterial strains and culture conditions were obtained from ATCC.

Peptide synthesis. Peptides were synthesized by Fmoc-based solid-phase peptide synthesis on Rink Amide ProTide resin. 1M Oxyma and 0.5M N,N'-Diisopropylcarbodiimide (DIC) were used for activation and the synthesis was carried out in a Liberty Blue Automated Microwave Peptide Synthesizer (CEM, Matthews, NC). AMP2 and AMP3 were synthesized with 0.1M N, NDiisopropylethylamine (DIEA) in the Oxyma solution and 1M DIC. Resin cleavage and sidechain deprotection was performed under argon using a trifluoroacetic acid:thioanisole:1,2ethanedithiol:anisole (90:5:3:2) solution while stirring for 2 hours (AMP1), 3 hours (AMP2, AMP3) and 3 hours at $30^{\circ} \mathrm{C}$ (MAD1). Crude peptide was subsequently precipitated with cold $\left(4^{\circ} \mathrm{C}\right)$ diethyl ether and isolated by centrifugation at $5000 \mathrm{rpm}$ for 5 minutes. Diethyl ether was decanted and sample lyophilized overnight.

Peptides were purified by reverse-phase HPLC (Shimadzu, Columbia, MD) equipped with a Phenomenex Semi-Prep Luna Omega PS C18 column (Torrance, CA). Analytical LCMS solvents consisted of solvent $A(0.1 \%$ formic acid in water) and solvent $B$ ( $9: 1$ acetonitrile and water with $0.1 \%$ formic acid). Preparative HPLC solvents consisted of solvent A $(0.1 \%$ 
trifluoroacetic acid in water) and solvent B (9:1 acetonitrile and water with $0.1 \%$ trifluoroacetic acid). A linear gradient of $0-25 \%$ solvent B over 25 minutes followed by $25-45 \%$ solvent B over an additional 40 minutes was used to purify D/L-MAD1 and AMP2. A linear gradient of 0-45\% solvent B over 45 minutes was used to purify AMP1. A linear gradient of 0-25\% solvent B over 15 minutes followed by $25-45 \%$ solvent B over an additional 40 minutes was used to purify AMP3. After lyopholization to collect pure peptide, all compounds were characterized by LC-MS ESI (+) to define purity (Supplementary Figs. 11 - 15).

Bacterial growth. Mycobacterium tuberculosis (H37Ra) and Mycobacterium smegmatis (Mc2 155) were cultured in Middlebrook $7 \mathrm{H} 9$ broth supplemented with $0.05 \%$ (v/v) polysorbate 80 , $0.5 \%(\mathrm{v} / \mathrm{v})$ glycerol and $10 \%(\mathrm{v} / \mathrm{v})$ oleic acid-albumin-dextrose-catalase (OADC). Methicillin resistant Staphylococcus aureus (MRSA; USA300), Methicillin sensitive Staphylococcus aureus (MSSA; NRS72), Klebsiella pneumoniae (NCTC 9633), Pseudomonas aeruginosa (POA-1) and Acinetobacter baumannii (ATCC 19606) were cultured in cation adjusted Mueller-Hinton broth (CAMHB), Bacillus anthracis (34F2) was cultured in lysogeny broth (LB). Haemophilus influenzae (ATCC 49247) was cultured in brain heart infusion (BHI). All cultures were grown at $37^{\circ} \mathrm{C}$ in a shaking incubator $(200 \mathrm{rpm})$, as advised by the Clinical and Laboratory Standards Institute (CLSI).

Liposomal membrane preparation. Liposomes were formed using extrusion techniques as previously described. ${ }^{34,35}$ In brief, Gram-positive, Gram-negative and mammalian model membranes were prepared by combining appropriate combinations of lipids in chloroform to a final concentration of $5 \mathrm{mM}$. Lipid solution was then dried under a stream of argon, and film lyophilized overnight to dry completely. The film was rehydrated with $2 \times$ concentrated liposome buffer (300 mM BTP, $100 \mathrm{mM} \mathrm{NaF,} \mathrm{pH} \mathrm{7.4).} \mathrm{The} \mathrm{solution} \mathrm{was} \mathrm{then} \mathrm{extruded} \mathrm{through} \mathrm{an} \mathrm{Avanti}$ Mini Extruder (Alabaster, Alabama) with a $0.8 \mu \mathrm{m}$ nuclepore polycarbonate membrane $>11$ times. For Gram-positive membranes, a mixture of DOPG and CL (58:42) was used to mimic Staphylococcus aureus. ${ }^{36}$ For Gram-negative membranes, a mixture of DOPE:DOPG:CL (65:23:12) was used to mimic Pseudomonas aeruginosa. ${ }^{37}$ For mammalian membranes, a mixture of POPC:DOPE (3:1) was used to model a typical lung epithelial cell. ${ }^{38}$ For mycomembrane models, Trehalose-6,6-dimycolate (TDM) was solubilized at $2.5 \mathrm{mg} / \mathrm{mL}$ in $10 \%$ n-Octyl- $\beta$-D-glucopyranoside (OG) as a surfactant and vortexed. The solution was then extruded through an Avanti Mini Extruder (Alabaster, Alabama) with a $0.1 \mu \mathrm{m}$ nuclepore polycarbonate membrane $>11$ times. Following extrusion, all liposome formulations were dialyzed against $10 \mu \mathrm{M}$ phosphate buffer for 24 hours using 300 kDa MWCO dialysis tubing. Dynamic light scattering (DLS) was performed to ensure uniform particle sizes for each liposomal composition (Supplementary Fig. 16).

Minimum inhibitory concentration determination. Bacterial growth was monitored by performing optical density (OD) readings at $600 \mathrm{~nm}\left(\mathrm{OD}_{600}\right)$. Triplicate 2-fold serial dilutions of each treatment were made in strain appropriate media and added to a 96-well microtiter plate. Bacteria were diluted to an $\mathrm{OD}_{600}$ of 0.002 and added directly to the treated wells in a $1: 1$ volume ratio. The microtiter plates were incubated at $37^{\circ} \mathrm{C}$, and bacterial growth was assessed by visual evaluation. Treatment lasted 24 hours for MRSA, MSSA, $K$. pneumoniae, $P$. aeruginosa, $A$. baumannii, $B$. anthracis and $H$. influenzae, 48 hours for $M$. smegmatis, and 96 hours for $M$. tuberculosis, as determined by doubling time. The minimum inhibitory concentration (MIC) was determined by observing the lowest concentration at which the 
compound prevented significant growth as compared to the untreated control. Assays were repeated on at least 3 separate genetic populations $(n \geq 9)$, and the lowest repeatable MIC is reported.

Circular dichroism. CD analysis was performed using a J-1500 Circular Dichroism Spectrometer (JASCO, Oklahoma City, OK). In a typical experiment, $20 \mu \mathrm{M}$ peptide in $10 \mathrm{mM}$ phosphate buffer at $\mathrm{pH} 7.4$ was added to a $10 \mathrm{~mm}$ path length quartz cell and wavelength spectra collected from 180 to $260 \mathrm{~nm}$ at $25^{\circ} \mathrm{C}$. Molar ellipticity $[\theta]$, measured in ( $\left.10^{3} \cdot \mathrm{deg} \cdot \mathrm{C} \mathrm{m}^{2} \cdot \mathrm{dmol}^{-1}\right)$, was calculated from the following expression: $\operatorname{mdeg} /(10 \cdot \mathrm{L} \cdot \mathrm{C})$ where mdeg is measured ellipticity in millidegrees, $L$ is path length in centimeters, $C$ is molar concentration. For pH-dependent CD studies, MAD1 was dissolved at $20 \mu \mathrm{M}$ in $\mathrm{pH}$ adjusted phosphate buffer. Temperature-dependent $\mathrm{CD}$ was performed at $\mathrm{pH} 4.5$ by increasing the temperature from $0^{\circ} \mathrm{C}$ to $90^{\circ} \mathrm{C}$ at 5 degree intervals, allowing 3 minutes of equilibration before measurements were taken. The reciprocal ramp down was subsequently performed with a similar step interval.

For liposomal CD studies, liposome solutions were diluted to a total lipid concentration of $240 \mu \mathrm{M}$ in phosphate buffer before mixing with an equal volume of $40 \mu \mathrm{M}$ MAD1. CD wavelength spectra were measured from 180 to $260 \mathrm{~nm}$ at $37^{\circ} \mathrm{C}$ in a $10 \mathrm{~mm}$ path length quartz cell. Measurements were performed immediately after addition of peptide, every 5 minutes until 30 minutes after addition, every 15 minutes until 60 minutes after treatment and every 30 minutes thereafter. The solution was mixed by pipette between each measurement. In a paired experiment, the same sample preparation was used, with $\mathrm{OD}_{600}$ measurements being collected. Time points were set at every minute for 15 minutes, every 5 minutes until 30 minutes, and every 30 minutes thereafter. All CD measurements were performed with an average of three replicates.

All-atom Discrete Molecular Dynamics simulations. A complete description of the DMD algorithm can be found elsewhere. ${ }^{39,40}$ Briefly, DMD uses discrete step function potentials to model particle-particle interactions instead of continuous functions used in traditional molecular dynamics. This discretization allows instantaneous change in velocity upon collision of particles according to the conservation of energy, momentum, and angular momentum. Implementation of a collision table to track possible collisions, shortening simulation time step to $50 \mathrm{fs}$, from 2 fs common in traditional molecular dynamics. These innovations significantly decrease computational time, allowing us to achieve a significant increase in sampling. We use an allatom protein model, ${ }^{41}$ which explicitly represents all heavy atoms and polar hydrogens, using infinite square-well constraints to represent bond lengths, angles, and dihedrals. Non-bonded interactions are adapted from the Medusa force field, ${ }^{42}$ van der Waals interactions are modeled using the Lennard-Jones potential, solvation is modeled by the Lazaridis-Karplus solvation model ${ }^{43}$ and hydrogen bonding interactions are modeled by the reaction algorithm. ${ }^{44}$

Model systems. The linear MAD1 peptide was created using PyMol (Shrödinger), and allowed to fold in equilibrium simulations at temperature 0.55 (monomer) or 0.4 (pentamer) $\mathrm{kcal} /\left(\mathrm{mol} \mathrm{k} \mathrm{k}_{\mathrm{B}}\right.$ ) ${ }^{1}$ for 100 ns. Pentamer simulations were initialized with randomized relative orientations of the five folded peptides, with no initial contact. Systems were simulated at constant volume with periodic boundary conditions in a 100-angstrom cubic box. Acidic pH (5.5) was modeled by protonation of histidine residues. 
Replica exchange. We used the replica exchange method to perform parallel simulations of the simulation system at multiple temperatures spanning the melting curve of the protein. ${ }^{45,46} \mathrm{At}$ intervals of $5 \mathrm{ps}$, replicas of neighboring temperatures exchange temperature values according to the Metropolis criterion, increasing sampling efficiency by increasing ability to overcome energetic barriers. For MAD1 monomer simulations, 10 replicas were used at temperatures of $0.330,0.360,0.400,0.430,0.460,0.505,0.540,0.580,0.620$, and $0.640 \mathrm{kBT}$. For MAD1 pentamer simulations, 16 replicas were used at temperatures of $0.340,0.360,0.380,0.400$, $0.420,0.440,0.460,0.480,0.500,0.520,0.540,0.560,0.580,0.600,0.620$, and $0.640 \mathrm{kcal} /(\mathrm{mol}$ $\left.\mathrm{k}_{\mathrm{B}}\right)^{-1}$. Each replica simulation is performed for $100 \mathrm{~ns}$, for a total of $1 \mu \mathrm{s}$ (monomer) or $1.6 \mu \mathrm{s}$ (pentamer).

WHAM analysis and identification of structural transition states. We combine replica trajectories using the MMTSB tool ${ }^{47}$ for weighted histogram analysis method (WHAM) for analysis of folding dynamics. ${ }^{48}$ WHAM computes the density of states by combining histograms from overlapping simulation trajectories. We calculate potential energy distributions over each simulation using intermediate temperatures so as not to introduce structural aberrations in representative structures from low- and high-temperature conditions. We identified three peaks and/or shoulders in the distribution, and identify the energy of each state as the histogram peak. We identify representative structures of each state by clustering structures whose energies fall within $5 \mathrm{kcal} / \mathrm{mol}$ around the peak by RMSD using the OC suite, ${ }^{49}$ with RMSD cutoff as the global maximum in a histogram of pairwise RMSDs of all structures clustered. The centroid of the largest cluster is chosen as the representative structure of that state.

Interface contact maps. In each simulation snapshot, we evaluate pairwise contacts between all residues in the simulation. We define two residues as being in contact if their $\mathrm{C}_{\beta}\left(\mathrm{C}_{\alpha}\right.$ for glycine) are within 7.5 angstroms of each other. The count between every pair of residues is normalized for the total number of simulation snapshots.

Electron microscopy. Transmission electron micrographs of diluted samples were obtained using a Tecnai G2 Spirit BioTwin transmission electron microscope (FEl; Hillsboro, OR) with 80 kV accelerating voltage by a side-mounted Gatan Orius SC200 CCD camera. Fibrils imaged by TEM were prepared as follows. MAD1 was solubilized at $20 \mu \mathrm{M}$ in phosphate buffer at either $\mathrm{pH}$ 4.5 or 7.4 and incubated at room temperature for 30 minutes. Then, $5 \mu \mathrm{L}$ was deposited on a Formvar/carbon coated 400 mesh $\mathrm{Cu}$ grid and allowed to adsorb for 5 minutes. This solution was then wicked away and the grid was washed twice with buffer. In the same manner, $3 \mu \mathrm{L}$ of $0.2 \mu \mathrm{m}$ filtered $1 \%$ uranyl acetate was deposited on the sample as a negative stain to enhance image contrast and immediately wicked until dry.

Mtb was diluted to an $\mathrm{OD}_{600}$ of 0.01 and treated with MAD1 at $0.1 \times, 0.5 \times, 1 \times$, and $5 \times \mathrm{MIC}$, and incubated at $37^{\circ} \mathrm{C}$ for 30 minutes. Following treatment, cells were pelleted by centrifugation at $7000 \mathrm{rpm}$ for 10 minutes at $4^{\circ} \mathrm{C}$. After overnight pre-fixation with a $2.5 \%$ glutaraldehyde, 0.1 $\mathrm{M}$ sodium cacodylate buffer, the bacterial cells were washed twice and then post-fixed with $1 \%$ osmium tetroxide for 2 hours. The fixed samples were subsequently washed twice with PBS and dehydrated for 15 minutes in each of a graded ethanol series (50,70,90, and 100\%) along with for 10 minutes in both a mixture $(1: 1, v / v)$ of absolute ethanol and acetone, and absolute acetone. Subsequently, the samples were transferred to a mixture $(1: 1, \mathrm{v} / \mathrm{v})$ of absolute acetone and epoxy resin for 30 minutes followed by pure epoxy resin for overnight at a constant temperature. Finally, the specimens were sectioned using an ultramicrotome (Leica EM UC6 
Microtome; Wetzlar, Germany), double stained by uranyl acetate and lead citrate, and observed by TEM.

For scanning electron microscopy studies, bacterial samples were prepared as above and imaged with a Zeiss SIGMA VP-FESEM (Oberkochen, Germany) with a $10 \mathrm{kV}$ landing voltage. Samples were prepared by pelleting MAD1-treated cells via centrifugation at 7000 rpm for 10 min. at $4^{\circ} \mathrm{C}$ and washed twice with PBS under similar conditions. After overnight pre-fixation with $2.5 \%$ glutaraldehyde and $0.1 \mathrm{M}$ sodium cacodylate buffer, samples were washed thrice with PBS. The fixed samples were subsequently dehydrated for $5 \mathrm{~min}$. in each of a graded ethanol series $(25,50,70,85,95$, and $3 \times 100 \%)$. Afterwards, the samples were transferred to a critical point dryer (Leica EM CPD300; Wetzlar, Germany), where they were subjected to slow $\mathrm{CO}_{2}$ exchange during 14 addition cycles at $28^{\circ} \mathrm{C}$ and 1 bar. Finally, the specimens were mounted onto aluminum stubs with conductive tabs, sputter coated with $10 \mathrm{~nm} \mathrm{Au/Pd}$ and observed by SEM.

Surface Plasmon Resonance. MAD1-peptidoglycan binding kinetics were determined using surface plasmon resonance spectroscopy (SR7500DC, Reichert Analytical Instrument; Depew, NY) using a method previously reported, ${ }^{50,51}$ with minor modification. In particular, a carboxylfunctionalized sensor chip was used to immobilize $S$. aureus derived peptidoglycan, and the running buffer was $10 \mathrm{mM}$ phosphate. In a typical experiment the surface was activated with a solution of $0.05 \mathrm{~g} / \mathrm{mL}$ EDC and $0.02 \mathrm{~g} / \mathrm{mL}$ NHS for 10 minutes at $20 \mu \mathrm{L} / \mathrm{min}$. For immobilization, $3 \mu \mathrm{g} / \mathrm{mL}$ peptidoglycan in $10 \mathrm{mM}$ phosphate buffer $(\mathrm{pH}=7.4)$ was injected at $5 \mu \mathrm{L} / \mathrm{min}$, followed by $1 \mathrm{mM}$ ethanolamine at $10 \mu \mathrm{L} / \mathrm{min}$ to block unbound activated sites. A second activation, immobilization and blockage cycle was run with peptidoglycan at $10 \mu \mathrm{g} / \mathrm{mL}$. The instrument was equilibrated with running buffer for $30 \mathrm{~min}$. before experiments. For binding analyses, MAD1 was flowed over the chip at $25 \mu \mathrm{L} / \mathrm{min}$ for $6 \mathrm{~min}$., followed by running buffer to obtain the dissociation profile. The biosensor was regenerated between treatment conditions with $40 \mathrm{mM}$ $\mathrm{NaOH}$ at $100 \mu \mathrm{L} / \mathrm{min}$ for $1 \mathrm{~min}$. Data was collected using SPR Autolink Software (Reichert Technologies) and plotted using Scrubber 2.0 (BioLogic Software). Concentration data were zeroed to baseline by subtracting the reference channel signal. Kinetic analysis was performed with a 1:1 biomolecular interaction model fit, which enables the association $\left(k_{a}\right)$ and dissociation $\left(k_{d}\right)$ constants to be determined and for the binding affinity $\left(K_{D}\right)$ to be calculated.

Membrane disruption. 1-N-phenylnaphthylamine (NPN) uptake was assessed in a Molecular Devices Spectramax i3 plate reader $\left(\lambda_{\mathrm{ex}}=340 \mathrm{~nm}, \lambda_{\mathrm{em}}=425\right)$. M. tuberculosis $(\mathrm{H} 37 \mathrm{Ra})$ cultures were grown to an $\mathrm{OD}_{600}$ of 1.0 and diluted to a final $\mathrm{OD}_{600}$ of 0.01 in unsupplemented Middlebrook 7H9 media to minimize background fluorescence caused by NPN interaction with hydrophobic components. Bacterial suspensions were treated with MAD1 at $0.1 \times, 0.5 \times, 1 \times, 2 \times$ and $5 \times$ MIC. $75 \mu \mathrm{L}$ of $2 \times$ treatment and bacterial suspensions were added to a round bottom multiwell plate with $n \geq 5$, and incubated for $30 \mathrm{~min}$. Following incubation, cells were pelleted out via centrifugation at $7000 \mathrm{rpm}$ for $10 \mathrm{~min}$ at $4^{\circ} \mathrm{C}$. $100 \mu \mathrm{L}$ of $10 \mu \mathrm{M}$ NPN was added to an opaque black multiwell plate. $100 \mu \mathrm{L}$ of supernatant from the treatment plate was added and incubated 5 minutes before measurement. Results were normalized to relevant background controls and calculated as a ratio with respect to the untreated control.

Impedance spectroscopy. The IS measurements were performed using HP Agilent 4192A LF impedance analyzer connected to a set of two interdigitated electrodes (IDE). The IDEs were fabricated using photolithography techniques with electrodeposition of nickel on a glass 
substrate, as detailed elsewhere. ${ }^{28}$ For each experiment, $100 \mu \mathrm{L}$ of the target solution is deposited over the sensor. A $30 \mathrm{mV}$ AC voltage is applied between the IDEs and the frequency is swept from $2 \mathrm{kHz}$ to $5 \mathrm{MHz}$. We studied three different sample conditions: baseline media, culture supernatants and cells transferred to an equal volume of fresh medium $\left(O D_{600}=0.1\right)$. In order to elucidate the effect of the peptide on Mtb cells, each condition is further split between peptide-treated and non-peptide-treated samples (negative control samples), for a total of six sample conditions. After testing of each solution, the sensor is rinsed with Milli-Q Ultrapure deionized (DI) water and blow-dried with clean,dry air for consistency. At each frequency, the impedance can be expressed as $Z=i Z \vee e^{j \theta}$, where $\quad \dot{Z V \vee i}$ is the impedance magnitude and $\theta$ is the its angle. We have utilized changes in $i Z \vee i$ as the basis for these analyses, as calculated by $\Delta Z=100 *\left(\dot{\zeta} Z \vee-\left[\left.|Z|\right|_{\text {baseline }}\right) /\left[\left.|Z|\right|_{\text {baseline }}\right.\right.$. Three droplets were analyzed, and one droplet was analyzed thrice, for an $\mathrm{n}$ of 5 for each condition, to ensure interand intra-droplet consistency.

Polymicrobial assays. Mtb, S. aureus, and K. pneumoniae were each back diluted to an $\mathrm{OD}_{600}$ of 0.004 in $7 \mathrm{H} 9$ broth and mixed in equal volumes. Polymicrobial cultures were then treated with MAD1 at various concentrations relative to the MIC of the peptide against Mtb. Treatment, or media control, was added to a final total bacterial $\mathrm{OD}_{600}$ of 0.003 . The co-culture was incubated for an hour, pelleted by centrifugation at $7000 \mathrm{rpm}$ for 10 minutes at $4^{\circ} \mathrm{C}$ and washed four times in PBS and resolubilized in $10 \%$ of the original volume. $100 \mu \mathrm{L}$ were dried and fixed on a glass slide, before staining with auramine-rhodamine following manufacturer's instructions. Samples were imaged using an Olympus IX73 microscope (Tokyo, Japan). Three images each were taken of 3 samples and analyzed by Image J software. 15 cells per image were analyzed ( $n=$ 135) for cellular fluorescence, with standard error reported.

In parallel, peptide cytotoxicity curves are generated for each of the tested species (mycobacteria, Gram-positive and Gram-negative). Treatment was performed in the same manner described for MIC assays. Mtb plates were grown for 7 days to reach an adequate $\mathrm{OD}_{600}$. Following incubation, wells are thoroughly mixed via pipette and transferred to a flat bottom plate for optical density measurements. Treatments were performed with $n>3$. Curve fitting was performed using GraphPad Prism software as a nonlinear regression of semi log data.

For growth inhibition studies, cultures at an $\mathrm{OD}_{600}$ of 0.001 were treated with MAD1 at $4 \times \mathrm{MIC}_{\mathrm{Mtb}}$ and growth monitored via $\mathrm{OD}_{600}$ measurements over time. Untreated cultures were monitored as controls. OD $_{600}$ measurements for Mtb were taken every 3 days, while $S$. aureus and $K$. pneumoniae were monitored every 2-4 hours until cultures reached stationary phase. The resulting data were analyzed using nonlinear sigmoidal regression as performed by GraphPad Prism. All curves were performed at $n=12$ and include at least 2 genetic populations.

Cell toxicity. NL20 and HUVEC were plated in tissue-treated 96-well plates at $5 \times 10^{3}$ cells/well, and allowed to adhere overnight. THP-1 monocytes were plated at $1.5 \times 10^{4}$ cells/well in a 96well plate and incubated for 72 hours in media supplemented with 200 nM PMA to induce differentiation into naive $\mathrm{MO}$ macrophages, as previously described. ${ }^{52}$ Following differentiation, THP-1 cells were incubated overnight in PMA-free media. All cells were treated for 24 hours with serial dilutions of MAD1. Cells treated with blank media or 20\% DMSO served as negative and positive controls, respectively. After treatment, cells were washed with blank media before addition of $100 \mu \mathrm{L}$ MTT solution $(0.5 \mathrm{mg} / \mathrm{mL})$ and incubated for $2 \mathrm{~h}$. Supernatant was removed 
and the cell impermeable formazan product was solubilized by the addition of $100 \mu \mathrm{L}$ DMSO and absorbance read with a microplate reader at $540 \mathrm{~nm}$. Percent viability was calculated with the following equation: $\begin{gathered}A \dot{i}-A b s_{\text {pos }} \\ \left(A b s_{\text {treatment }}-A b s_{\text {pos }}\right) / i\end{gathered}$. The resulting data were analyzed using nonlinear regression of semi log data as performed by GraphPad Prism. All curves were performed at $\mathrm{n}=12$ and include at least 2 genetic populations.

Combinatorial drug screening for synergy. Checkerboard assays were performed by preparing 2-fold, $4 \times$ serial dilutions of the peptide in media and added to a 96-well microtiter plate. 2-fold, $4 \times$ serial dilutions of each TB antibiotic was prepared in a separate 96-well microtiter plate in an orthogonal direction to the peptide. The antibiotic dilutions were then added to their respective rows in the plate of diluted peptide in a 1:1 volume ratio. As with MIC assays, $50 \mu \mathrm{L}$ of bacterial solutions at an $\mathrm{OD}_{600}$ of 0.002 were added to all wells for a final assay volume of $100 \mu \mathrm{L}$. The plate was sealed and incubated at $37^{\circ} \mathrm{C}$ for 4 days before growth determined via visual inspection.

Statistical Analysis. Statistical significance was determined by performing a two-tailed, unpaired student's t-test using GraphPad Prism software, unless stated otherwise. A confidence level of $95 \%(\alpha=0.05)$ was used. Significance denoted in figures is denoted as: ${ }^{*} p<0.01$ and ${ }^{* *} p<0.001$, unless exact $p$ value is given.

Data Availability. The data that support the findings of this study are available from the corresponding author upon reasonable request.

Code Availability. The parallelized Discrete Molecular Dynamics simulation engine ( $\pi D M D$, v1.0) with Medusa all-atom force field is available from Molecules In Action, LLC (free to academic users, moleculesinaction.com). 


\section{References}

1 Blaser, M. J. Antibiotic use and its consequences for the normal microbiome. Science $\mathbf{3 5 2}$, 544-545 (2016).

2 Brito, I. L. et al. Mobile genes in the human microbiome are structured from global to individual scales. Nature 535, 435-439 (2016).

3 Smillie, C. S. et al. Ecology drives a global network of gene exchange connecting the human microbiome. Nature 480, 241 (2011).

4 Levy, M., Blacher, E. \& Elinav, E. Microbiome, metabolites and host immunity. Curr. Opin. Microbiol. 35, 8-15 (2017).

5 Levy, M., Kolodziejczyk, A. A., Thaiss, C. A. \& Elinav, E. Dysbiosis and the immune system. Nat. Rev. Immunol. 17, 219 (2017).

6 Brown, E. D. \& Wright, G. D. Antibacterial drug discovery in the resistance era. Nature 529, 336-343 (2016).

7 Melander, R. J., Zurawski, D. V. \& Melander, C. Narrow-Spectrum Antibacterial Agents. Medchemcomm 9, 12-21 (2018).

8 Niederweis, M. Mycobacterial porins - new channel proteins in unique outer membranes. Mol. Microbiol. 49, 1167-1177 (2003).

9 Mahfoud, M., Sukumaran, S., Hülsmann, P., Grieger, K. \& Niederweis, M. Topology of the porin MspA in the outer membrane of Mycobacterium smegmatis. J. Biol. Chem. 281, 5908-5915 (2006).

10 Alderwick, L. J., Harrison, J., Lloyd, G. S. \& Birch, H. L. The Mycobacterial Cell WallPeptidoglycan and Arabinogalactan. Cold Spring Harbor Perspect. Med. 5 (2015).

11 Vandal, O. H., Pierini, L. M., Schnappinger, D., Nathan, C. F. \& Ehrt, S. A membrane protein preserves intrabacterial $\mathrm{pH}$ in intraphagosomal Mycobacterium tuberculosis. Nat. Med. 14, 849-854 (2008).

12 Vergne, I. et al. Mechanism of phagolysosome biogenesis block by viable Mycobacterium tuberculosis. Proc. Natl. Acad. Sci. 102, 4033-4038 (2005).

13 Deretic, V. et al. Mycobacterium tuberculosis inhibition of phagolysosome biogenesis and autophagy as a host defence mechanism. Cell. Microbiol. 8, 719-727 (2006).

14 Cochran, A. G., Skelton, N. J. \& Starovasnik, M. A. Tryptophan zippers: Stable, monomeric ß-hairpins. Proc. Natl. Acad. Sci. 98, 5578-5583 (2001).

15 Liu, J., Yong, W., Deng, Y., Kallenbach, N. R. \& Lu, M. Atomic structure of a tryptophanzipper pentamer. Proc. Natl. Acad. Sci. 101, 16156-16161 (2004).

16 Heinz, C., Karosi, S. \& Niederweis, M. High-level expression of the mycobacterial porin MspA in Escherichia coli and purification of the recombinant protein. J. Chromatogr. B: Biomed. Sci. Appl. 790, 337-348 (2003).

17 Ragazzon, G. \& Prins, L. J. Energy consumption in chemical fuel-driven self-assembly. Nat. Nanotechnol. 13, 882-889 (2018).

18 Lin, Y. et al. Residue-Specific Solvation-Directed Thermodynamic and Kinetic Control over Peptide Self-Assembly with 1D/2D Structure Selection. ACS Nano 13, 1900-1909 (2019).

19 Vandal, O. H., Nathan, C. F. \& Ehrt, S. Acid resistance in Mycobacterium tuberculosis. J. Bacteriol. 191, 4714-4721 (2009).

20 (ed The European Committee on Antimicrobial Susceptibility Testing) (2018). Pathogens Using Improved Synthetic Antibacterial Peptides. Antimicrob. Agents Chemother. 57, 2295-2303 (2013).

22 Brown, L., Wolf, J. M., Prados-Rosales, R. \& Casadevall, A. Through the wall: extracellular vesicles in Gram-positive bacteria, mycobacteria and fungi. Nat. Rev. Microbiol. 13, 620630 (2015). 
23 Maitra, A. et al. Cell wall peptidoglycan in Mycobacterium tuberculosis: An Achilles' heel for the TB-causing pathogen. FEMS Microbiol. Rev. 43, 548-575 (2019).

24 Muheim, C. et al. Increasing the permeability of Escherichia coli using MAC13243. Sci. Rep. 7, 17629 (2017).

25 Helander, I. \& Mattila-Sandholm, T. Fluorometric assessment of Gram-negative bacterial permeabilization. J. Appl. Microbiol. 88, 213-219 (2000).

26 Eriksson, M., Nielsen, P. E. \& Good, L. Cell permeabilization and uptake of antisense peptide-peptide nucleic acid (PNA) into Escherichia coli. J. Biol. Chem. 277, 7144-7147 (2002).

27 Halder, S. et al. Alteration of Zeta potential and membrane permeability in bacteria: a study with cationic agents. Springerplus 4, 672-672 (2015).

28 Butler, D., Goel, N., Goodnight, L., Tadigadapa, S. \& Ebrahimi, A. Detection of bacterial metabolism in lag-phase using impedance spectroscopy of agar-integrated 3D microelectrodes. Biosens. Bioelectron. 129, 269-276 (2019).

29 Bolotsky, A. et al. Two-Dimensional Materials in Biosensing and Healthcare: From In Vitro Diagnostics to Optogenetics and Beyond. ACS Nano 13, 9781-9810 (2019).

30 Inoue, S. et al. Dielectrophoretic characterization of antibiotic-treated Mycobacterium tuberculosis complex cells. Anal. Bioanal. Chem. 407, 7673-7680 (2015).

31 Perera, A. S., Wang, H., Shrestha, T. B., Troyer, D. L. \& Bossmann, S. H. Nanoscopic surfactant behavior of the porin MspA in aqueous media. Beilstein J. Nanotechnol. 4, 278284 (2013).

32 Truant, J., Brett, W. \& Thomas Jr, W. Fluorescence microscopy of tubercle bacilli stained with auramine and rhodamine. Henry Ford Hosp. Med. J. 10, 287-296 (1962).

33 Danilchanka, O., Pavlenok, M. \& Niederweis, M. Role of Porins for Uptake of Antibiotics by Mycobacterium smegmatis. Antimicrob. Agents Chemother. 52, 3127-3134 (2008).

34 Medina, S. H. et al. An Intrinsically Disordered Peptide Facilitates Non-Endosomal Cell Entry. Angew. Chem., Int. Ed. 55, 3369-3372 (2016).

35 Rath, P. et al. Cord factor (trehalose 6,6'-dimycolate) forms fully stable and non-permeable lipid bilayers required for a functional outer membrane. Biochim. Biophys. Acta, Rev. Biomembr. 1828, 2173-2181 (2013).

36 Epand, R. F., Savage, P. B. \& Epand, R. M. Bacterial lipid composition and the antimicrobial efficacy of cationic steroid compounds (Ceragenins). Biochim. Biophys. Acta, Biomembr. 1768, 2500-2509 (2007).

37 Lombardi, L. et al. Antimicrobial peptides at work: interaction of myxinidin and its mutant WMR with lipid bilayers mimicking the P. aeruginosa and E. coli membranes. Sci. Rep. 7, 44425-44425 (2017).

38 Zehethofer, N. et al. Lipid Analysis of Airway Epithelial Cells for Studying Respiratory Diseases. Chromatographia 78, 403-413 (2015).

39 Dokholyan, N. V., Buldyrev, S. V., Stanley, H. E. \& Shakhnovich, E. I. Discrete molecular dynamics studies of the folding of a protein-like model. Folding Des. 3, 577-587 (1998).

40 Proctor, E. A., Ding, F. \& Dokholyan, N. V. Discrete molecular dynamics. Wiley Interdiscip. Rev.: Comput. Mol. Sci. 1, 80-92 (2011).

41 Ding, F., Tsao, D., Nie, H. \& Dokholyan, N. V. Ab initio folding of proteins with all-atom discrete molecular dynamics. Structure 16, 1010-1018 (2008).

42 Ding, F. \& Dokholyan, N. V. Emergence of protein fold families through rational design. PLoS Comput. Biol. 2, e85 (2006).

43 Lazaridis, T. \& Karplus, M. Effective energy function for proteins in solution. Proteins: Struct., Funct., Bioinf. 35, 133-152 (1999).

44 Ding, F., Borreguero, J. M., Buldyrey, S. V., Stanley, H. E. \& Dokholyan, N. V. Mechanism for the $\alpha$-helix to $\beta$-hairpin transition. Proteins: Struct., Funct., Bioinf. 53, 220-228 (2003). 
45 Sugita, Y. \& Okamoto, Y. Replica-exchange molecular dynamics method for protein folding. Chem. Phys. Lett. 314, 141-151 (1999).

46 Okamoto, Y. Generalized-ensemble algorithms: enhanced sampling techniques for Monte Carlo and molecular dynamics simulations. J. Mol. Graphics Modell. 22, 425-439 (2004).

47 Feig, M., Karanicolas, J. \& Brooks III, C. L. MMTSB Tool Set: enhanced sampling and multiscale modeling methods for applications in structural biology. J. Mol. Graphics Modell. 22, 377-395 (2004).

48 Kumar, S., Rosenberg, J. M., Bouzida, D., Swendsen, R. H. \& Kollman, P. A. The weighted histogram analysis method for free-energy calculations on biomolecules. I. The method. J. Comput. Chem. 13, 1011-1021 (1992).

49 Barton, G. OC-A cluster analysis program. University of Dundee, Scotland, UK (2002).

50 Coyne, J., Davis, B., Kauffman, D., Zhao, N. \& Wang, Y. Polymer Microneedle Mediated Local Aptamer Delivery for Blocking the Function of Vascular Endothelial Growth Factor. ACS Biomater. Sci. Eng. 3, 3395-3403 (2017).

51 Burch, J. M., Mashayekh, S., Wykoff, D. D. \& Grimes, C. L. Bacterial Derived Carbohydrates Bind Cyr1 and Trigger Hyphal Growth in Candida albicans. ACS Infect. Dis. 4, 53-58 (2018).

52 Xie, Z. et al. Immune Cell-Mediated Biodegradable Theranostic Nanoparticles for Melanoma Targeting and Drug Delivery. Small 13, 1603121 (2017). 
